\title{
Fluorescence Microscopy: Established and Emerging Methods, Experimental Strategies, and Applications in Immunology
}

\author{
HOWARD R. PETTY ${ }^{1,2 *}$ \\ ${ }^{1}$ Department of Ophthalmology and Visual Sciences, University of Michigan Medical School, Ann Arbor, Michigan 48105 \\ ${ }^{2}$ Department of Microbiology and Immunology, University of Michigan Medical School, Ann Arbor, Michigan 48105
}

\section{KEY WORDS fluorescence; microscopy; imaging}

\begin{abstract}
Cutting-edge biophysical technologies including total internal reflection fluorescence microscopy, single molecule fluorescence, single channel opening events, fluorescence resonance energy transfer, high-speed exposures, two-photon imaging, fluorescence lifetime imaging, and other tools are becoming increasingly important in immunology as they link molecular events to cellular physiology, a key goal of modern immunology. The primary concern in all forms of microscopy is the generation of contrast; for fluorescence microscopy contrast can be thought of as the difference in intensity between the cell and background, the signal-to-noise ratio. High information-content images can be formed by enhancing the signal, suppressing the noise, or both. As improved tools, such as ICCD and EMCCD cameras, become available for fluorescence imaging in molecular and cellular immunology, it is important to optimize other aspects of the imaging system. Numerous practical strategies to enhance fluorescence microscopy experiments are reviewed. The use of instrumentation such as light traps, cameras, objectives, improved fluorescent labels, and image filtration routines applicable to low light level experiments are discussed. New methodologies providing resolution well beyond that given by the Rayleigh criterion are outlined. Ongoing and future developments in fluorescence microscopy instrumentation and technique are reviewed. This review is intended to address situations where the signal is weak, which is important for emerging techniques stressing super-resolution or live cell dynamics, but is less important for conventional applications such as indirect immunofluorescence. This review provides a broad integrative discussion of fluorescence microscopy with selected applications in immunology. Microsc. Res. Tech. 70:687-709, 2007. @ 2007 Wiley-Liss, Inc.
\end{abstract}

\section{INTRODUCTION}

Experimental immunology relies heavily upon fluorescence tools such as fluorochrome-based enzyme assays, flow cytometry, and fluorescence microscopy. The key advantage of fluorescence tools is their inherently greater sensitivity and range in comparison to methods based upon changes in optical density or chemiluminescent emission; the latter process emits one photon per molecule in comparison to hundreds to thousands of photons emitted by one fluorochrome. In the past twenty years, flow cytometry has contributed greatly to the development of immunology including such diverse areas as the characterization of cell populations and the selection of hybridomas. Flow cytometry tells us a little about a great many cells whereas the complementary tool of fluorescence microscopy tells us a great deal about only a comparatively few cells. Flow cytometry, however, is not very informative concerning the spatial locations or dynamics of constituent molecules and cannot be extended to in vivo experiments. For these and other reasons, fluorescence microscopy has been of growing importance in immunology. Broad-based developments over the past twenty years, such as confocal microscopy and two-photon fluorescence microscopy, have contributed greatly to the structural analysis of immune cells and tissues. The impact of fluorescence microscopy is poised to skyrocket. Just a few current or ongoing fluorescence microscopy contributions now possible are:

- Single molecule studies of individual proteins,

- Fluorescence fluctuation correlation spectroscopy and fluorescence resonance energy transfer to study molecular associations in living cells,

- Use of quantum dots to perform single molecule studies in living cells,

- In vivo trafficking of immune cells or targets using fluorescent labels,

- Total internal reflection microscopy to explore single channel opening events, and

- Greatly improved resolution using stimulated emission depletion microscopy, $4 \mathrm{Pi}$ imaging and structured illumination.

*Correspondence to: Dr. Howard R. Petty, Department of Ophthalmology and Visual Sciences, 1000 Wall Street, The University of Michigan Medical School, Ann Arbor, MI 48105, USA. E-mail: hpetty@umich.edu

Received 18 December 2006; accepted in revised form 31 January 2007

Contract grant sponsors: NCI and Wilson Medical Foundation.

DOI 10.1002/jemt.20455

Published online 28 March 2007 in Wiley InterScience (www.interscience. wiley.com). 
The available tools, such as fluorescence microscopy instrumentation, drive experimental immunology, cell biology, and biochemistry. As these tools increase in number over the next few years, our knowledge of biological mechanisms will improve.

Of the microscopy tools recently developed, much commercial attention has been focused upon high sensitivity detectors. However, it is not just the detector's sensitivity, but the signal-to-noise ratio of the entire apparatus including the computational analysis that determines image characteristics; hence one must address sources of noise and their management. This is particularly true because new high numerical aperture objectives (NA = 1.45-1.65) are much better at collecting both signal and noise in an experiment. The suppression of noise begins with the laboratory setting and ends with image processing methods. In this review I discuss strategies to improve the detection of weak fluorescence signals by enhancing the signal and suppressing noise. Practical laboratory suggestions are outlined. I also review background information relevant to fluorescence microscopy, the current state of the field, especially as it applies to immunology, and current and future developments. The various forms of fluorescence microscopy, such as conventional widefield, scanning confocal, total internal reflection imaging, etc., are discussed. Much of the practical advice focuses upon conventional epi-fluorescence imaging, but most of this is applicable to other forms of fluorescence microscopy.

\section{ADVANTAGES OF MICROSCOPIC STUDIES}

Optical microscopy methods enjoy many advantages over other technologies. (1) Experiments are generally conducted under physiological conditions. As living cells are examined at $37^{\circ} \mathrm{C}$, chemical fixation and de-

\begin{tabular}{ll}
\hline & \\
CCD & \multicolumn{1}{c}{ ?Abbreíiations } \\
CMOS & charge coupled device \\
DSP & dithio-bis(succinimidyl propionate) \\
EGFP & enhanced green fluorescent protein \\
EMCCD & electron-multiplying CCD \\
FITC & fluorescein isothiocyanate \\
FLIM & fluorescence lifetime imaging microscopy \\
FP & fluorescent proteins \\
FRET & fluorescence resonance energy transfer \\
GFP & green fluorescent protein \\
ICCD & intensified CCD \\
I 2 i & image interference micrscopy \\
IR & infrared \\
LED & light emitting diodes \\
LUT & look-up-table \\
MCP & multichannel plate \\
NSOM & near-field scanning optical microscopy \\
NA & numerical aperture \\
SSIM & structured-illumination fluorescence microscopy \\
STED & stimulated emission depletion \\
TIRF & Total internal reflection fluorescence \\
$\lambda$ & wavelength \\
&
\end{tabular}

TABLE 1. Survey of fluorescence microscope techniques

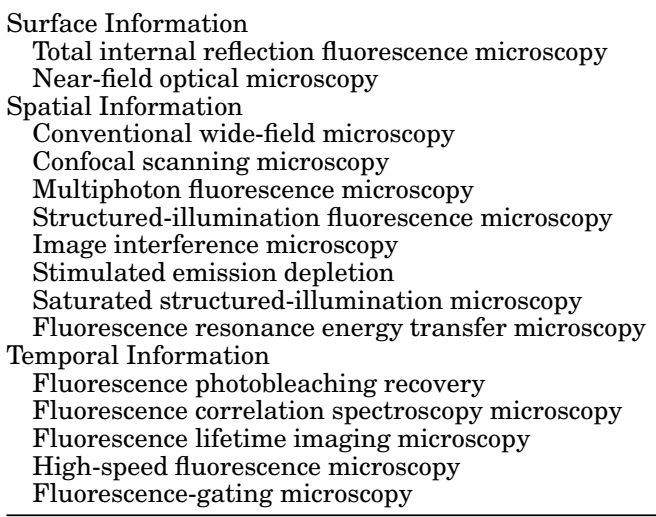

tergent solubilization are unnecessary, thus minimizing their attendant artifacts. (2) Cells can be selected based upon morphological criteria (thereby excluding contaminating or dead cells) or the presence of ongoing cell-cell interactions. (3) The readout is instantaneous, as information is traveling at the speed of light. (4) The approach is more efficient in terms of the amount of material required. (5) Under certain circumstances individual molecules can be observed. (6) Results are not measured as population averages, thus retaining spatiotemporal information. (7) Experiments can be extended to in vivo conditions. (8) Spatial resolution limits defined previously by the wave properties of light have been breached, thus bringing optical microscopy ("nanoscopy") closer to the realm of electron microscopy. 9) Optical methods exhibit great flexibility in design and application (Table 1). Because of these advantages and their emerging applications, the use of optical tools in immunology is expected to grow at an increasing rate.

\section{RESOLUTION The Rayleigh Criterion}

The wave properties of light impose some restrictions, or at least difficulties, on the resolution provided by optical microscopy. As originally pointed out by Abbe (1873), the resolution of optical microscopy may be limited by diffraction. The apparent size of a single molecule or point is greatly increased by this effect. The change in an object from a point to its inflated microscopic appearance is referred to as the point spread function. The amount of diffraction inflation depends upon the wavelength of light and the finite size of the microscope's objective. Now, if we have two molecules far apart on an uncrowded uniform substrate, we can tell that there are two molecules present, although they are inflated by diffraction, but are nonetheless resolved from one another. When two molecules get close to one another, it becomes more difficult to tell if there are one or two molecules present. Several criteria have been established to make this determination, such as the Sparrow criterion and the Rayleigh criterion. Specific criteria may be more relevant to specific experimental situations or set of assumptions, but here we will only consider the familiar Rayleigh criterion. For situations where the Rayleigh criterion applies, 
the lateral resolution ( $\mathrm{x}-\mathrm{y}$ plane) is given by:

$$
d_{\mathrm{x}, \mathrm{y}}=0.61 \lambda / \mathrm{NA}
$$

where $\lambda$ is the wavelength and $\mathrm{NA}=$ the numerical aperture. The NA is given by $n \sin \Theta / 2$ where $n$ is the refractive index of the medium between the objective and sample (e.g., immersion oil) and $\Theta / 2$ is the half-angle cone of the light captured by the objective. The axial resolution ( $z$ or microscope optical axis) is:

$$
d_{\mathrm{z}}=2 \lambda / \mathrm{NA}^{2}
$$

These equations are appropriate for epi-fluorescence microscopy wherein the objective also serves as the condenser, but for white light illumination the equations need to be re-written to account for the difference in numerical apertures of the condenser and objective. In conventional wide field microscopy, the lateral resolution is generally about $200 \mathrm{~nm}$ and the axial resolution is roughly $600-800 \mathrm{~nm}$. It is important to remember that these are observational criteria under a narrow set of assumptions, not physical laws. Fundamentally, it is Heisenberg's Uncertainty Principle, not the Rayleigh criterion, which governs a microscope's resolution. Consequently, it is possible to extract information beyond these traditional limits.

\section{Survey of Strategies to Improve Resolution}

A number of options are now available to enhance fluorescence microscope images based upon altering the type of microscope used or the treatment of images. In early experiments, Allen and Inoue developed video microscopy, where adjustments to the gain and offset of the signal are used to improve the appearance of a wide field image (Inoue, 1989; Inoue and Inoue, 1989). These adjustments may be performed before or after image acquisition (e.g., video or digital). Although the concepts behind confocal microscopy were developed by the early $1960 \mathrm{~s}$, practical applications were not seen until the 1980s. Confocal's primary benefit was improved axial resolution. Another widely available scanning method, two-photon microscopy, also provides enhanced resolution. Similar improvements in resolution can be obtained using wide field illumination coupled with z-scanning and computational deconvolution of images. Improvements can also be obtained using commercial structured-illumination systems for conventional wide field microscopes. These improvements are significant, but not dramatic. Stimulated emission depletion fluorescence microscopy, a new fluorescence microscope technique that is not yet generally available, promises to increase resolution by 10 -fold in comparison to Rayleigh's criterion. Moreover, it is generally assumed that a signal-to-noise ratio $>1$ is necessary to extract information from images. This conventional wisdom has also been supplanted by new computational processing methods, such as wavelet analysis. I will now discuss several technical aspects of these tools.

\section{SUMMARY OF FLUORESCENCE MICROSCOPES}

Fluorescence

A molecule or atom can absorb electromagnetic energy thereby promoting it into a higher electronic, vibrational, and/or rotational state. As a rule, only energy in specific amounts corresponding to prescribed energy level differences can be absorbed by a molecule. This is why water, which absorbs microwave radiation, warms in a microwave oven, but a microwave does not directly warm ceramic or plastic containers as they have no rotational levels matching the energy levels of microwaves. Usually, radiation absorbed by a sample is dissipated as heat. A very small number of molecules known as fluorophores ${ }^{1}$ absorb specific wavelengths of electromagnetic energy to be promoted to a higher electronic energy state followed by the emission of longer wavelength light known as fluorescence (Lakowicz, 1983). This feature of higher energy absorption and lower energy emission, which is known as the Stokes' shift, is one property of fluorescence. Another characteristic physical feature of fluorescent molecules is the lifetime of their excited state. Fluorescent lifetimes are generally in the vicinity of $10 \mathrm{~ns}$, but this often varies between 1 and 100 ns. In general, a fluorophore's excited state is more polar than its ground state (Lakowicz, 1983). One can visualize this as follows: when an electron is promoted to a higher energy level it is further away from the remainder of the molecule thereby creating a more asymmetric or polar charge distribution. This change in a fluorophore's polarity with excitation leads to solvent effects in which its physical properties are dependent upon solvent. As the excited state is more polar than the ground state, it takes more energy (lower wavelength) to excite a molecule in a hydrophobic environment such as a membrane than in an aqueous environment such as the cytosol. Consequently, both the excitation and emission spectra will be effected by the solvent conditions. The fluorescence lifetime can be changed by solvent conditions as the more polar excited state is stabilized in a polar environment. All of these physical features-the intensity of fluorescence, spectral changes, and lifetime changes can be exploited in fluorescence microscopic studies to better understand molecular and cellular processes.

\section{Fluorescence Microscopes}

As there are many types of fluorescence microscopes, it is useful to outline a few of the basic features of these instruments. I will begin by considering the way in which excitation light is delivered to the sample, which is often used to define the type of fluorescence microscope (Fig. 1).

Wide-Field and Structured Illumination. Conventional wide-field fluorescence microscopes should be familiar to all readers. Conventional fluorescence microscopy is performed with a compound microscope that includes an objective lens and eyepieces. During wide-field imaging, the entire sample is illuminated with excitation light and can be viewed through the oculars. The resolution of fluorescence micrographs obtained using conventional fluorescence microscopy

\footnotetext{
${ }^{1}$ Fluorophores or fluorochromes are chromophores that exhibit fluorescence.
} 


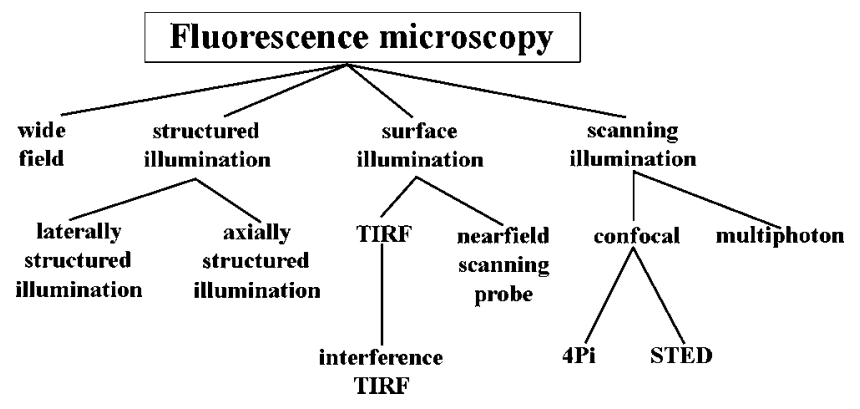

Fig. 1. Relationships among fluorescence microscopes. One manner in which to understand the relationships among fluorescence microscopies is by classification according to sample illumination. In this figure, fluorescence microscopy is divided into four major groups: wide field, structured illumination, surface illumination, and scanning illumination.

can be enhanced using computational deconvolution techniques, which remove "haze" and correct for an instrument's point spread function.

Using a wide-field fluorescence microscope, further improvements in resolution can be made using structured illumination. In this case a ruling (or interfering laser illumination) is used to excite fluorescence. When a ruling is placed in the back focal plane of a microscope, a series of excitation stripes are projected in the plane of the sample ( $x-y$ plane). This is laterally structured illumination. Of course, just one picture provides less information than conventional wide field imaging. However, in this method three images are collected with the ruling in three different positions. In addition to the data contained in conventional wide-field images, additional higher resolution information in the form of moiré fringes is contained in the data set. The data set is then mathematically analyzed to extract image information encoded in the "virtual" moiré pattern. The primary advantage to this approach is the improvement in lateral resolution, although some improvement in axial resolution is also provided (Gustafsson, 1999, 2000; Neill et al., 1997).

In addition to laterally structured illumination, it is also possible to axially structure the excitation light ( $z$-axis). This approach is based on the interference properties of light waves. This method was pioneered by Lanni's laboratory (Balle et al., 1993) and stimulated the development of other interference-based fluorescence microscopy methodologies (see below). A sample is illuminated in a wide-field mode with two unfocused counter-propagating laser beams. This light interferes to form a standing wave that varies throughout a sample along the $z$-axis. High axial resolution information is contained in the image, which is extracted by analyzing images acquired at three different phases. As not all spatial frequencies are sampled by this approach, some spatial information in the sample may be missed.

Surface Illumination. The methods described above provide illumination in all three dimensions of a sample. In addition to these three-dimensional illumination schemes, tools have also been developed that allow the specific illumination of a two-dimensional surface. Total internal reflection fluorescence (TIRF) microscopy, first introduced by Axelrod and colleagues, is one such method (Axelrod, 2001). This approach utilizes a conventional wide-field fluorescence microscope, but the excitation light is delivered to the sample so that it undergoes total internal reflection at the interface between the sample and substrate. Typically, a prism or high NA objective is needed to promote total internal reflection. Although the light undergoes total internal reflection, an evanescent electromagnetic field is found adjacent to the substrate. As the intensity of the field decays exponentially with distance from the surface, only a very small layer $(<100 \mathrm{~nm})$ of the sample adjacent to the substrate is illuminated, which eliminates most of the background fluorescence. Although this provides high axial resolution, it is only applicable to single molecules, cell surface components, and other thin layers. Nonetheless, there are many interesting immunological events involving receptors, ion channels opening events (Demuro and Parker, 2005), exocytosis (Ober et al., 2004), and lipid rafts taking place at surfaces. Recently, interference effects have been used to create patterned TIRF excitation light, which further increased the lateral resolution of this method (Cappello et al., 2003).

Another technique that provides surface excitation is near-field scanning optical microscopy (NSOM) (Lewis et al., 2003). Although the detection elements of a NSOM system are similar to that of wide-field microscopes discussed above, the excitation strategy is quite different. In NSOM, an optical probe with an aperture of $20-100 \mathrm{~nm}$ is brought to within $5-50 \mathrm{~nm}$ of a surface. A small fraction of the light entering the optical probe exits from the aperture to illuminate the sample. The probe is scanned across the surface much like that of scanning tunneling or atomic force microscopy. In contrast to conventional fluorescence microscopy, $\mathrm{d}_{\mathrm{x}, \mathrm{y}}$ depends upon the tip's aperture, not the $\lambda$ of light. Maximum excitation is provided at the illumination point, with little contribution from points $\sim 30 \mathrm{~nm}$ away from the tip. Lateral resolution is thereby enhanced greatly. As the excitation light rapidly spreads out with distance from the tip, there is little out-of-focus fluorescence. Although it is possible to use NOSM on aqueous samples, it is not easy. Most studies have examined biological structures such as chromosomes, but cellular studies are beginning to appear in the literature. For example, Koopman et al. (2004) have imaged single molecules on the surface of dendritic cells.

Scanning Illumination. The two best-known scanning methods in fluorescence microscopy are scanning confocal microscopy and multiphoton microscopy. In scanning confocal microscopy, a laser is used to form a spot that is scanned back-and-forth across a sample in two-dimensions to form an image. As each image is assembled pixel-by-pixel, scanning methods are inherently slow compared to wide field methods where all pixels are simultaneously measured. Additionally, the quantum efficiencies of CCD detectors used in wide field imaging are higher than the detectors used in confocal imaging. A pinhole is placed in front of the detector; in-focus emission light is passed to the detector whereas out-of-focus emission is blocked. If the pinhole is much smaller than the spot, a condition that is infrequently met, a 1.4-fold improvement in lateral resolution will be obtained. Discrimination in the axial 
dimension is improved by a factor of about two, depending upon instrument attributes such as spot and pinhole size.

Another approach to increase axial resolution is to utilize multi-photon excitation. During two-photon microscopy, a sample is illuminated with light that has one-half the energy needed to excite the dye. By using focused laser light of high intensity, two photons can be simultaneously absorbed by one dye molecule, which then emits its characteristic light. As absorption is proportional to the intensity squared and the intensity varies along the $z$-axis, light just above and just below focus cannot be excited and therefore cannot contribute to the image. This provides another physical means of removing out-of-focus fluorescence from images, although no gain in lateral resolution occurs unless it is combined with confocal imaging. As long wavelength light penetrates tissues to greater depths, two-photon imaging can probe further into tissues. The disadvantage is that an expensive ultrafast laser is needed to reach the required intensities and that these high intensities may damage living cells.

In addition to the types of fluorescence microscopy outlined above, it is also possible in some instances to combine these various methods, such as scanning confocal and multiphoton microscopy. In addition, it is also possible to cross illumination boundaries; for example, interference TIRF is both a type of surface illumination and structured illumination microscopy. The more specialized emerging methods of STED and 4Pi imaging will be discussed later.

Fluorescence Lifetime Imaging Microscopy. The fluorescence techniques described above rely upon detecting the emission intensity in the spectral region of the fluorophore under study. Alternatively, it is possible to measure the differences in fluorescence lifetime from pixel-to-pixel to image this distinct physical parameter; this approach is called fluorescence lifetime imaging microscopy (FLIM) (Suhling et al., 2005; Wallrabe and Periasamy, 2005). One chief application of FLIM is fluorescence resonance energy transfer (FRET) microscopy. Of course, there are other ways of detecting FRET, such as the excitation and emission characteristics of the fluorophores. Indeed, it is advisable to confirm FRET using more than one physical measure. There are several experimental methods to perform FLIM. Broadly, one can illuminate a sample with pulses of excitation light or the light can vary in a sinusoidal fashion. In the pulsed excitation mode, a scanning microscope can illuminate a pixel and the decay kinetics directly measured (time domain). Using a system with a time-varying light source, the properties of the emission light such as its phase can be used to deduce the dye's lifetime (frequency domain). In both approaches data are analyzed by computer to calculate the lifetime image. The advantage of using a pulsed laser is that the data are richer in information, allowing multiple decay components to be resolved. The advantage of the frequency domain measurements is that these instruments are substantially less costly. FLIM has been used to study TCR complex assembly and the immune synapse (Treanor et al., 2005; Zal and Gascoigne, 2004), antigen presentation (French et al.,
1997), and CD44 interaction with cytoskeletal regulatory proteins (Legg et al., 2002).

\section{LABORATORY SETTING}

Fluorescence microscopes to be used at low light levels must be set-up in appropriate darkrooms. The microscope facility should be carefully prepared, just as one might do for a conventional photographic darkroom. For example, the doors and joints near pipes in the ceiling should be light tight. Walls can be painted flat black to minimize reflections. As these facilities have limited space and high current usage equipment, such as mercury lamps and lasers, supplemental air conditioning may be required to meet OHSA standards. Furthermore, careful temperature control is important because the performance of computers and electronics is temperature-sensitive. Modern fluorescence microscopy laboratories generally have higher power requirements than typical research facilities. The power requirements can be divided into clean power and dirty power. Dirty power is the electrical supply for lamps, lasers, or other utilities that have high current pulses or noise. The key issue here is that dirty power could damage delicate electronics with or without a surge suppressor on the line. For example, I have a Zeiss temperature control device that can be damaged by turning on or off a nearby mercury lamp in the wrong order. As a rule, high current surge devices should be first on and last off during the day. In contrast, clean power is provided for cameras, computers, and similar devices with sensitive electronics. One can minimize potential coupling between circuits by placing them in different electrical panels on opposite sides of a building. The microscope should be placed on an optical table with easy access to electrical outlets.

\section{BRIEF SURVEY OF SIGNAL-TO-NOISE CONSIDERATIONS}

Because the refractive index of a cell is about equal to that of water, the generation of contrast, a difference in intensity between the region of interest and the background, is of paramount importance. This requirement promoted the development of cytochemistry, phase-contrast, differential interference contrast, fluorescence microscopy, and video-enhanced microscopy. There are many strategies to increase the contrast in fluorescence microscopy. One strategy to improve the detection of fluorescence signals is noise reduction. Another is to increase the brightness of a sample. As the brightness (B) of a fluorescent image is:

$$
B=\mathrm{NA}^{4} / \mathrm{mag}^{2}
$$

high NA objectives increase the brightness of a sample. For example, a NA $=1.65$ objective provides a 8 -fold brighter image than a NA $=1.0$ objective of the same magnification. For live cell labeling, it is important to use the highest amount of labeling possible without perturbing the cell responses under study. The label should have the highest dynamic range possible, not necessarily the brightest intensity, to yield the greatest contrast. The illumination source should provide the highest sample illumination with acceptable levels of 
photobleaching. The fluorescence should be captured using a detector with the highest gain and lowest noise available. Finally, data are processed to increase the apparent signal-to-noise ratio, with special consideration to the process under study, such as finding the edges of microtubules. However, one should never rely upon image processing tools to improve poorly designed experiments or those with inadequate attention to signal/noise factors. In the following paragraphs, I discuss these strategies in greater detail.

\section{MINIMIZING NOISE Sources of Unwanted Light}

With the exception of the fluorescence emission from the region of interest, all other photons reaching the detector constitute unwanted light and degrade the signal-to-noise ratio. As new detectors on the market can detect single photons, noise suppression becomes important at low light levels. Today's high NA objectives improve the collection of both signal and noise. Using a NA $=1.45$ objective, a 4 -fold improvement in brightness over older objectives was found, but noise also increases. Hence, the system's capacity to collect photons was improved, but little gain in signal-to-noise ratio or contrast was achieved. It is therefore important to insure that only the desired light is collected.

There are many potential sources of unwanted light. One important source of unwanted light is room light. Fluorescence microscopy is generally performed in a darkened room. However, light from computer monitors, lamps, LED equipment lights, etc. could all contribute to high ambient room light levels, especially if there are several microscopes in the same room.

Another source of unwanted light is the microscope. One of the microscope's sources of unwanted light is irrelevant fluorescence emission. When a fluorochrome emits, it emits in all three-dimensional directions. Consequently, cells of no interest on the slide, which may or may not be visible in the field of view, will contribute to a sample's background light levels (haze). In addition to unwanted fluorescence emission, irrelevant epifluorescence illumination also contributes to noise. For example, most of the epifluorescence light illuminating a sample passes through the slide and coverslip. This light can be reflected from the white light condenser above the stage in an inverted scope back to the sample and is potentially a small source of noise. More broadly, all sources of epifluorescence illumination scattered in a microscope increase noise. One might think that this is unimportant because of the presence of an emission interference filter. However, interference filters are only effective for light traveling perpendicular to the filter. For example, if you tip an interference filter illuminated from behind, its color changes. Similarly, the wavelengths passed by an interference filter are a function of the angle of incidence (Pollack, 1966). Hence, light that would nominally not pass though the interference filter (the wrong wavelength) will pass through the filter when traveling off-axis. Light of the wrong wavelengths when scattered in the microscope can pass through the interference filter and may reach the detector. This can be a significant problem at low fluorescence emission levels. This is why, for example, the interior surfaces of microscope parts are black. Hence,

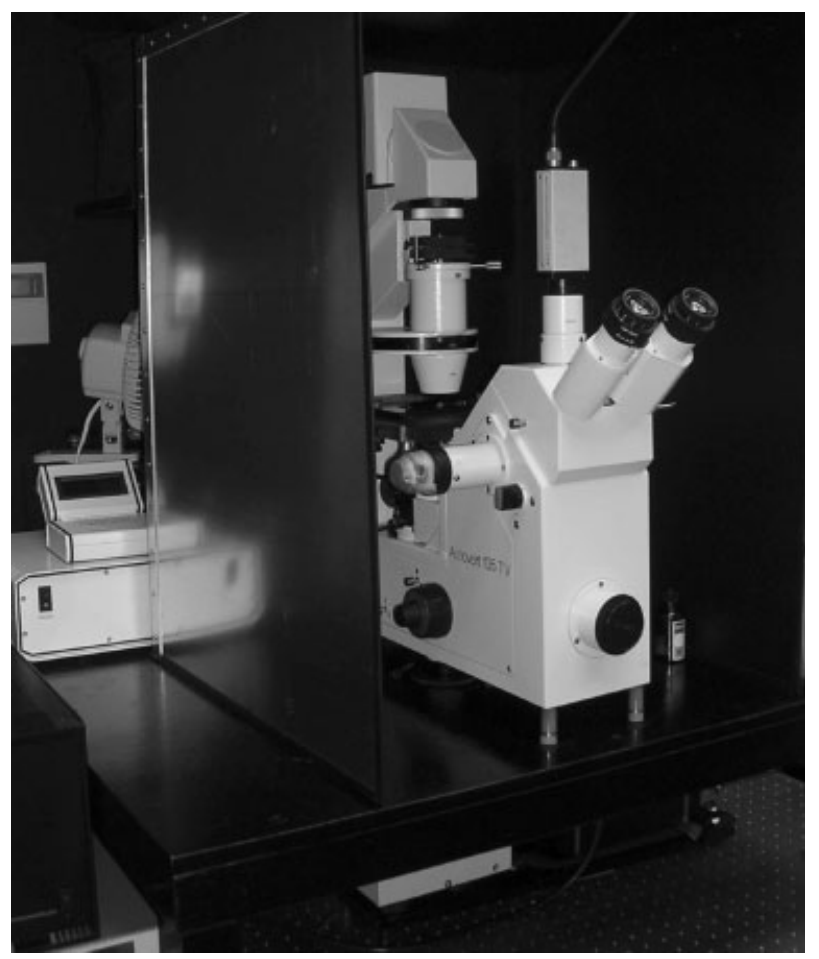

Fig. 2. Fluorescence microscope with an aluminum enclosure. The enclosure has a tight seal separating the microscope from the excitation source, which is behind the enclosure. The ICCD camera is attached to the bottom port, below the microscope table. The small CCD at the top port is used for bright field imaging. The black curtain can be lowered during data acquisition.

there are sources of noise that should be minimized when studying faint light sources.

\section{Reducing Unwanted Room Light}

There are many ways to stop room light from entering a microscope. The first and most obvious way to minimize room light noise is to close the ocular slider. Most microscopes have a lever for a slider that blocks light from entering the microscope through the eyepieces, which should always be closed during fluorescence imaging. Stray light can also enter a system through the objective. One way to reduce this is to place a 35-mm plastic film canister upside-down over the microscope slide. A hole is placed in the bottom of the film container to allow the excitation light to pass through (otherwise, the light would be reflected back into the sample). These two simple changes can significantly improve images.

Another useful strategy is to isolate the imaging portion of the microscope from the remainder of the lab. A microscope cover or enclosure can be constructed from aluminum to surround most of the scope (Fig. 2). The inside is painted flat black and black felt is attached to cover the front of the enclosure. When attached to a ground wire and a wire mesh-containing curtain, it also serves as a Faraday cage to protect interior electronics from stray electromagnetic fields. A rubber strip is attached to the aluminum to seal the space between the structure and the tube of the epifluores- 
cence attachment, which keeps stray lamp light away from imaging elements. Likewise, rubber strips may be placed on the front of the enclosure for safety. If heat within the enclosure is a concern, light-tight vents can be attached to the top. If air-flow near the $\mathrm{Hg}$ lamp is restricted, supplemental cooling may be necessary. This may be done by attaching a small clip-on fan to a nearby shelf or wall.

Several additional strategies can be used to improve signal detection. Computer monitors are one of the most serious contributors to stray room light. The impact of computer monitor light can be minimized in two ways: (1) some software programs will shut the monitor off during signal acquisition (for example, other labs have written subroutines for Princeton Instruments software that carry out this function), and (2) computer monitor privacy filters can be used to reduce peripheral stray light from reaching the microscope. Microscopes should be checked for light leaks. If light leaks are found on the body of the microscope (uncommon under most conditions for new microscopes), they can be blocked with electrical tape from the outside (inelegant, but effective). Common areas for light leaks are sites where surfaces meet. This includes points of attachment of cameras, etc. Black felt can be used to help reduce light leaks at these sites, but care should be taken to avoid covering cooling ducts.

\section{Reducing Illumination Noise}

Illumination noise is a major source of error in many quantitative fluorescence microscopy studies. By illumination noise, I mean all sources of noise contributing to fluctuations in lamp intensity, including the bulb and power supply, as well as spatial variations in lamp intensity. Illumination noise will not be a serious issue if only qualitative information is necessary, such as the location of a protein. Lamp fluctuations are less important when using longer periods of image acquisition due to averaging of the illumination light (although drift noise will still be present) or image ratioing (see Image Ratioing Microscopy). However, if quantitative comparisons in the intensities of two micrographs or photomultiplier tube readings are being made, it may be useful to reduce the illumination noise of a system. The $p-p$ fluctuations of mercury lamp and power supply combinations provided by microscope manufacturers are typically $\pm 2.5 \%$ measured over several seconds (unpublished observations). Others have reported that xenon arc lamps, which are more stable than mercury lamps, have a $1-2 \%$ stability under the best of conditions (Breeze and Ke, 1972). Illumination noise is caused by arc point shift and flutter within the lamp. It is also caused by fluctuations in the current provided by the power supply. Under optimal conditions, the impact of lamp noise on fluorescence emission should be less than the shot noise level; for example, if $10^{6}$ counts are measured in a photometric experiment, then illumination noise should be less than $0.1 \%$. In an imaging experiment with 2,000 electrons collected in one pixel (1\% of the pixel depth of an Andor EMCCD), lamp noise remains greater than shot noise (2.2\%), provided that the lamp fluctuations have not been timeaveraged $(\sim 1 s)$. As $p-p$ illumination noise is on the order of $\mathrm{ms}$, illumination noise is a potential issue at video frame rates. If the shot noise and illumination noise are about equal with no other sources of error, the system noise will still be $40 \%$ greater than the shot noise alone. Hence, illumination noise reduction is often helpful in quantitative fluorescence microscopy.

There are several means of reducing the illumination noise of a microscope. Although tungsten-halogen lamps are inherently less noisy than mercury arc lamps, they are not discussed here because their emission spectra and illuminance make them less useful in fluorescence excitation. ${ }^{2}$ One means of reducing illumination noise is to employ light emitting diodes (LEDs) for fluorescence excitation. Recent studies have analyzed the noise associated with LED illumination sources and demonstrated their usefulness in biological studies (Nishimura et al., 2006; Rumyantsev et al., 2004 ) with fluctuations $<0.03 \%$. LED sources provide excellent monochromatic excitation. LEDs for microscopic work are now available commercially (Table 2).

Although LEDs have the advantage of low noise, they are only useful at a single wavelength. Using Hamamatsu's new superquiet xenon and mercury/xenon lamps, it is possible to substantially reduce the illumination noise in fluorescence microscopic and photometric work. Arc fluctuations are primarily due to variations in the number of electrons emitted by the cathode. ${ }^{3}$ The barium-impregnated cathodes of superquiet lamps minimize this effect; they also increase the bulb's lifetime to $\sim 1200 \mathrm{~h}$ due to the fact that the electrode erosion is eliminated. The temporal fluctuations of superquiet xenon lamps are roughly $0.2 \%$, which is far better than conventional light sources. It makes little sense to use these lamps in conventional power supplies exhibiting $2 \%$ ripple. ${ }^{4}$ Hamamatsu's power supply exhibits $0.1 \%$ ripple and drift, making it a good choice for use with the lamp. Newport has a power supply with $<1 \%$ noise that, when combined with a model 68950 light controller containing a thermoelectrically cooled PIN diode to monitor the lamp, reduces illumination noise to $<0.01 \%$. Thus, both passive noise suppression in the form of lamp construction and active noise suppression in the form of rapid current adjustment can be combined to minimize illumination noise. As illumination noise is a leading source of error in many quantitative applications, these strategies will be useful in making highly accurate measurements of immune cell signals.

In addition to the temporal noise described above, spatial noise may also accompany illumination. For example, laser illumination often exhibits spatial noise. Although incoherent sources such as lamps have less spatial noise, the illumination intensity may drop off at the edges of the field. Typically, this is not a major problem because digital cameras generally collect only a portion of the field. This is especially true if the field diaphragm is reduced in size to block unwanted emission light (see the following section). The mercury-cad-

\footnotetext{
${ }^{2}$ In contrast to arc lamps, the large filaments of tungsten-halogen lamps do not approximate point sources.

${ }^{3}$ Hamamatsu Inc., "Super-quiet mercury-xenon lamps." See: http://sales. hamamatsu.com/assets/pdf/catsandguides/lmps-Xe-HgXe.pdf

${ }^{4}$ In addition, lamp current and temperature may not be correct. Only power supplies and housings designed for a particular bulb should be used.
} 
TABLE 2. Representative suppliers of microscopes and accessories

\begin{tabular}{ll}
\hline Instrument/Material & \multicolumn{1}{c}{ Suppliers } \\
\hline $\begin{array}{l}\text { Conventional and } \\
\text { TIRF microscopes }\end{array}$ & Nikon, Zeiss, Olympus \\
$\begin{array}{l}\text { 4Pi Microscopes } \\
\text { Fluorescence lifetime } \\
\text { imaging microscopes }\end{array}$ & $\begin{array}{l}\text { Leica } \\
\text { TauTec, Lambert, Hamamatsu }\end{array}$ \\
$\begin{array}{l}\text { Structured illumination } \\
\text { microscopes }\end{array}$ & Zeiss \\
Structured illumination & Opti-Grid \\
$\quad$ add-on device & \\
Super-Quiet Xe and & Hamamatsu \\
$\quad$ Mercury-Xe lamps & Hamamatsu, Newport Corp. \\
Low noise power & \\
supplies for lamps & Newport Corp. \\
Oriel light controllers & \\
for lamps & Rapp, Andor \\
LED excitation sources & Andor, Photometrics \\
EMCCD cameras & Princeton Instruments \\
ICCD cameras & Optical Insights, \\
Multi-wavelength & Andor Inst.. \\
emission imaging systems & Photon Tech. Intl. \\
Software & Image J, Image-Pro, \\
High speed shuttering & Metamorph, AndorIQ \\
Flashlamps & Uniblitz, Sutter \\
Fluorescent probes & Perkin-Elmer, Hamamatsu, Rapp \\
Interference filters & Invitrogen (Molecular Probes), TefLabs \\
\hline & Omega Optical, Chroma \\
\hline
\end{tabular}

mium lamps of the X-cite system of EXFO-Lifesciences, Inc. have less intensity drop-off near the edges, but cannot match the temporal noise reduction afforded by the Hamamatsu and Newport systems. Spatial noise in illumination can be introduced by using fiber optic coupling to the microscope, as the number of fibers is finite. As liquid light guides produce less spatial illumination noise, they are becoming more popular in microscope illumination devices. It should also be possible to use circular holographic diffusers (Baturin et al., 2003) to reduce spatial illumination noise.

\section{Reducing Unwanted Light Originating From the Epi-Fluorescence Source}

The easiest way to reduce unwanted light from the epi-fluorescence source is to reduce the size of field diaphragm near the $\mathrm{Hg}$ lamp, as illustrated in Figure 3. Of course, the lamp must be properly set-up and the microscope adjusted for Köhler illumination first, then the field diaphragm should be stopped-down. This minimizes unwanted excitation and emission as well as unwanted scatter in the experiment.

Another obvious factor contributing to unwanted excitation light passing through to the detector is worn interference filters, especially excitation filters damaged by light. As the performance of interference filters declines over time (Meaburn, 1966), they must be periodically replaced. Indeed, as worn optical filters can cause severe eye damage, they should be routinely inspected visually. Damage can be detected by examining the filter using transillumination in a stereo microscope. If facilities are available, emission and excitation scans can be performed to verify filter performance. On the other hand, a new filter set can be used to ascertain the performance of an old set using standardized beads.
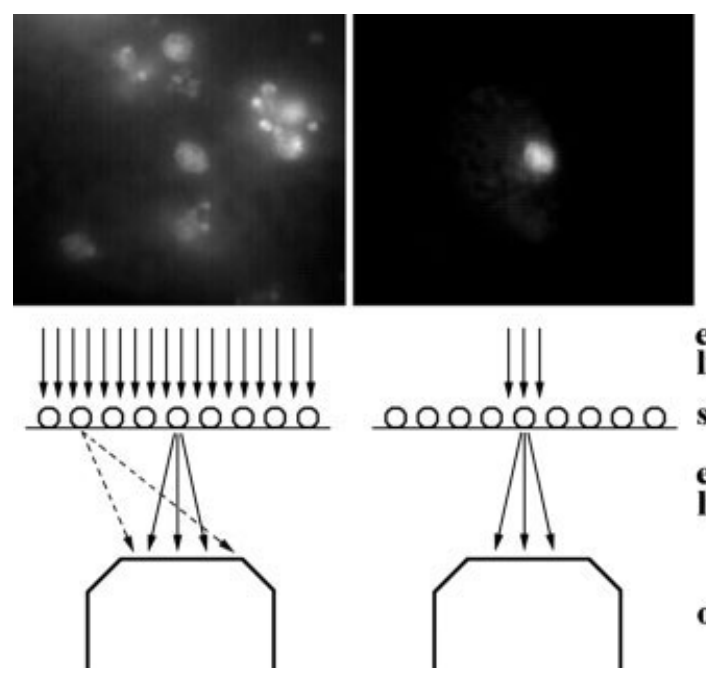

excitation light sample emission light

objective

Fig. 3. The benefits of field diaphragm adjustment. For both imaging and quantitative microfluorometry studies, the field diaphragm should be reduced in size. As fluorescence is emitted in all directions from a sample, light from irrelevant cells in the field of view as well as cells from outside the field of view may be captured by the objective. This effect is illustrated in the upper half of the figure. The lower half of the figure illustrates how cells outside the field of view contribute to the background haze.

In addition to unwanted room light, $\mathrm{Hg}$ and $\mathrm{Xe}$ lamps generate a lot of unwanted excitation wavelengths traveling through the microscope. Microscope manufacturers include an optical filter to block the IR component of this light. For example, Schott glass KG1 filters block $97 \%$ of the radiation at $1,000 \mathrm{~nm}$. A conservative estimate is that a $100 \mathrm{~W}$ lamp produces $\sim 10^{19}$ photons per second. A substantial fraction of this light is IR radiation. In general, CCD detectors are quite sensitive in the red and IR. Consequently, IR light must be managed properly. Most applications such as immunofluorescence microscopy and conventional lamp illumination are not likely to be seriously affected by IR, but IR haze is a potential problem with bright lamp sources and flashlamps. It is a potential source of haze in single molecule work. One means of removing UV and IR light from the illumination is to use a water filter; due to the difference in the absorption coefficient of water in the visible versus the UV and IR, these unwanted wavelengths of light can be removed at the epifluorescence source. To supply a real-world example, professional photographers use UV filters to reduce the background haze, invisible to their eyes but not conventional film, in their photographs. Similarly, microscopists need to be aware of this potential source of noise invisible to their eyes, but not CCDs.

One of the most useful contributions to noise reduction in microscopy in the past few years is the introduction of built-in light traps into microscopes on the illumination side of the sample. Light traps help separate signal and potential sources of noise (Herrero, 1992). These are now standard features in Zeiss and Nikon microscopes. Nikon reports a 5 -fold decrease in noise, a dramatic reduction that we have confirmed. The system removes unwanted excitation light from the opti- 


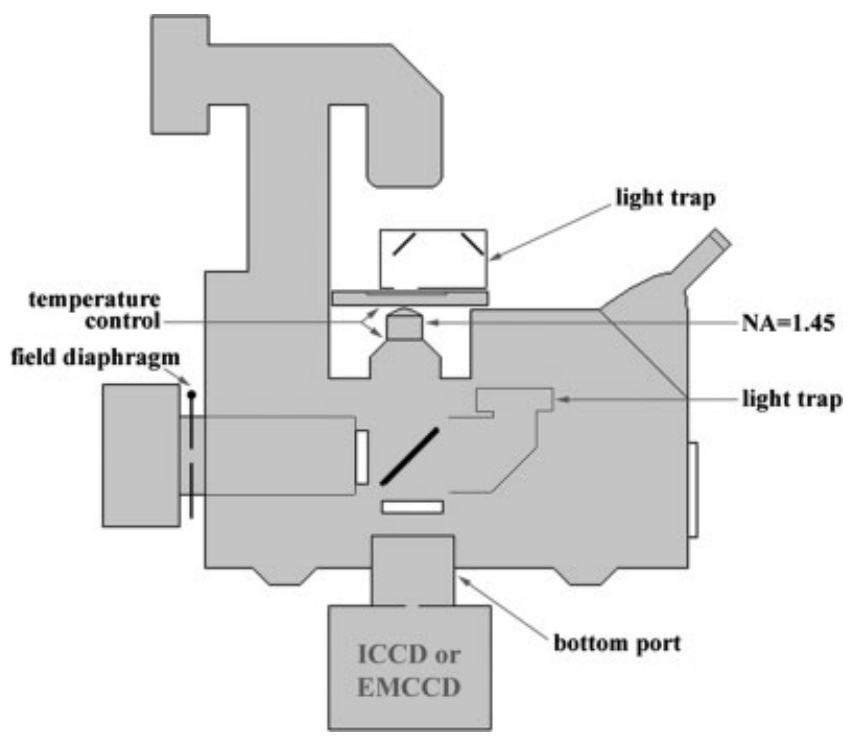

Fig. 4. Schematic diagram of an inverted microscope illustrating light traps. The latest models of Zeiss and Nikon microscopes have internal light traps that provide a dramatic 4 to 5 -fold improvement in the signal-to-noise ratio. Other factors that may improve the detection of weak fluorescence emission are high numerical aperture objectives, reduction of the field diaphragm, use of the bottom port, and use of an upper light trap, which is especially useful for crowded optical laboratories.

cal axis by allowing light not reflected by the dichroic mirror to pass into a light trap, which Nikon refers to as a noise terminator (schematically illustrated in Fig. 4). This light trap reflects and absorbs the light. For low light level work, light traps on the illumination side of the microscope are a clear advantage.

Light traps can also be added to the sample side. These are useful in removing unwanted epi-fluorescence light and keeping unwanted room light away from the objective. In this approach, a light-tight aluminum box with an off-axis hole to admit "waste" excitation light is used. The box is lined with black felt. A mirror with an attached neutral density filter is secured at the top of the box at a $45^{\circ}$ angle to the optical axis. Two of these are attached at each end of the box where they serve to absorb the light and remove it from the optical axis. The improvement with a light trap above the sample in an inverted microscope is very small in comparison to light traps on the excitation side, but may be useful if stray light is a problem.

\section{Reducing Unwanted Fluorescence Emission}

As described in the preceding section concerning epifluorescence excitation light, the simplest way to reduce unwanted fluorescence emission is to reduce the field diaphragm's size. As fluorescence from each point in a sample (fluorescence from the cell of interest and other cells) is emitted in three dimensions, regions of a sample separate from the cell of interest will contribute to the background noise or haze in the region of interest. By reducing the field diaphragm, this unwanted light is reduced. Similarly, plating cells at low density to increase intercellular distances also helps to alleviate this problem. This is needed for low light level studies. It is crucial in performing single cell quantitative microfluorometry studies because a large diaphragm will add nonspecific light to the specific light as well as decreasing the signal-to-noise ratio. For quantitative comparisons from day-to-day, it is useful to both monitor the field diaphragm's size and to have cells widely dispersed on a slide.

For studies near 350-450 nm, slides and cover-slips may contribute to background fluorescence. Some glass contains chromium color centers that increase the background fluorescence when excited in this region. Because of this potential complication, it is sometimes best to use quartz slides and cover-slips, as color centers are not found in quartz. Although this is not a problem for labeling levels such as those of conventional immunofluorescence, at low light levels this becomes important.

Another strategy to reduce unwanted light, such as a hot spot arising from reflections or scattering, is to insert a diaphragm or stop into the light path (Inoue and Spring, 1997). This may lead to vignetting, but vignetting will not be observed using digital cameras that capture only the central region of the field of view. In addition to managing these potential optical effects, this approach is also useful in imaging spectrophotometry (Zakrzewski, 2003).

Cell experiments are often conducted at $37^{\circ} \mathrm{C}$, as this is generally the physiologically relevant temperature. Another advantage of warmer temperatures is that the $K_{\mathrm{d}}$ of several calcium binding dyes significantly vary between room temperature and $37^{\circ} \mathrm{C}$. Although several benefits derive from performing experiments at $37^{\circ} \mathrm{C}$, one potential problem is density fluctuations of immersion oil. When a sample is kept at $37^{\circ} \mathrm{C}$ with the objective at room temperature, large fluctuations in the viscosity and, consequently, the refractive index of the immersion oil occur. Such variations invalidate TIRF and confocal experiments. This needlessly applies another layer of noise to experiments. This problem is solved by enclosing the entire system in an incubator or by using objective warmers to remove the temperature gradient.

\section{Time-Gated Imaging}

Another approach to manage unwanted light in fluorescence microscopy is time-gated imaging (Connally et al., 2002, 2004). This approach is illustrated diagrammatically in Figure 5. A brief pulse of light illuminates a sample for a short period of time. This light is provided using specialized lamps or lasers; in general, lasers operate at much higher repetition rates than lamps thereby increasing the rate of signal collection for short-lived fluorophores. Most fluorescent molecules have lifetimes between 1 and $100 \mathrm{~ns}$. By precisely turning the detector on-and-off at various times, one can gate the detector such that only fluorescence emission at specific times after the excitation pulse is collected. In this way autofluorescence, which is observed in this illustration at a shorter lifetime, is gated out of the fluorescence image, which is collected at longer times (Fig. 5). Although this is sometimes referred to as time-resolved fluorescence imaging, time is only being used to gate the detector. As time-gated imaging cannot resolve lifetimes, it should not be confused with FLIM. 


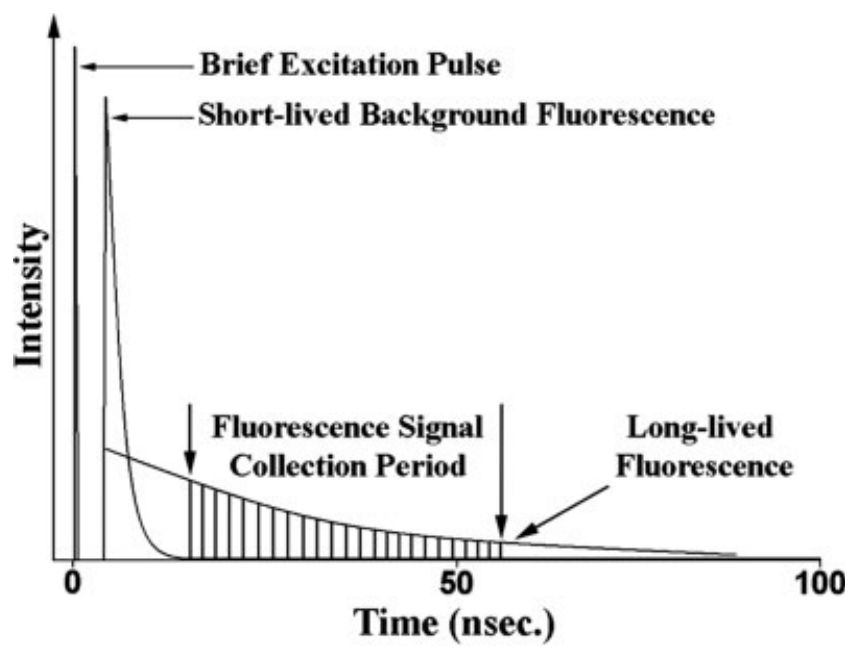

Fig. 5. The process of time-gated imaging is illustrated. The intensity is plotted at the ordinate while the abscissa gives the time. A sample is exposed to a brief pulse of excitation light. The sample molecules then emit their fluorescence with certain characteristic lifetimes. For many cell types, the nonspecific background fluorescence decays much more rapidly than the specific fluorescence emission. Operationally, one simply delays the detector's activation until much of the autofluorescence has decayed. If this process is repeated multiple times, one can accumulate high contrast images without the background autofluorescence.

In one application of time-gated imaging, microorganisms were detected in heterogeneous samples using a europium (Er)-labeled $\mathrm{Ab}$ directed against the organism. Er was used because it has a very long lifetime of $240 \mu \mathrm{s}$, which is much longer than the lifetime of autofluorescent contaminants. Depending upon a variety of factors, Connally et al. (2002, 2003, 2004) reported an 11- to 45 -fold improvement in signal-to-noise ratio by time-gating images. In addition to microbiological samples, complex eukaryotic samples have also been probed with time-gated imaging (Rulli et al., 1997). Other probes, including organic molecules have been used (de Hass et al., 1997). Instruments providing time-gated fluorescence imaging at relatively long lifetimes are commercially available. Although certain studies benefit greatly from time-gated imaging, it is not a widely used tool.

\section{Other Sources of Noise}

In addition to the sources of noise described above, several additional potential sources of noise should also be considered. Vibrations, which could arise from equipment or air ducts, are potential source of noise. A stable microscope is important. For example, weight lower to the table is better for stability; Zeiss microscopes are particularly well designed in this regard. Another source of noise is electromagnetic interference (EMI) due to other equipment. Faraday cages and conductive meshes can be used. In addition, coaxial cables should be used to minimize EMI. If ribbon cables are used, they can be surrounded by electrically conductive tape (3 M Corp.). Another potential source of fluorescence noise is contamination. When using cells grown in vitro, it is likely advantageous to avoid the use of dyes such as phenol red in the media. In addition, exog- enous contaminants such as color markers for white boards (which use fluorescent ink) can reduce microscope performance.

\section{MAXIMIZING SIGNAL Cleanliness}

The easiest step to take in preserving wanted light is to insure that the microscope is clean. On a regular basis, a system should be cleaned, adjusted, and repaired by a service representative from the manufacturer. Immersion objectives must be cleaned after every use according to the manufacturer's recommendations. ${ }^{5}$ The commercially available cleaner "Sparkle" (A.J. Funk, Elgin, IL) may be used to clean the objective, whereas all other commercially available glass cleaners are to be avoided (J. Stanciu, personal communication). Although lens paper may be appropriate for some microscopes, it may not be advisable in all situations.

\section{Objectives}

Objectives specifically designed for high performance fluorescence microscopy are available from most manufacturers, such as the Zeiss UltraFluar. These objectives transmit quite well in both the UV and visible regions of the spectrum (Piston, 1998). As illustrated in Eqs. (1) and (2), $d_{\mathrm{x}, \mathrm{y}}$ and $d_{\mathrm{z}}$ depend upon NA, not magnification. Because of their increased light collection ability and resolution, higher NA objectives are preferred for most fluorescence microscopy applications. Another issue in fluorescence microscopy is choosing an objective with the most appropriate magnification. According to the Nyquist criterion, the magnified lateral resolution $\left(d_{\mathrm{x}, \mathrm{y}}\right)$ should be spread over two pixels on the detector (Inoue and Spring, 1997). This can be expressed as:

$$
\text { Magnification }=2 \text { pixel size } / d_{\mathrm{x}, \mathrm{y}}
$$

For example, the optimum magnification for a microscope system comprised of a camera having a $6 \mu \mathrm{m}$ pixel size and objectives with NAs yielding $d_{x, y}=$ $0.2 \mu \mathrm{m}$ is $60 \times$. In this example, a $60 \times / 1.45$ objective would provide a magnification high enough to bring out all the detail in a sample, but low enough to provide the greatest brightness.

On the other hand, there may be occasions when a lower NA is preferred. For example, in quantitative microfluorometry using one or more photomultiplier tubes, it may be important to measure the intensity of a living cell independent of shape changes the cells may undergo. Salzberg et al. (1977) have shown that the measured fluorescence intensity of a cell is not significantly changed by moving the stage up or down by $50 \mu \mathrm{m}$ using a $20 \times / 0.4$ objective. As $d_{\mathrm{z}}$ is large, cell movements or errors in focus should not influence the output of photometric devices. The Zeiss $32 \times / 0.4$ objective is a reasonable compromise to increase the depth of field in these experiments, which is especially true as spatial resolution is unimportant. As indicated by

${ }^{5}$ http://www.zeiss.com/C1256D18002CC306/0/1205E8CBD68054EAC12570150 0404705/ \$file/46-0009_e.pdf. 
Eq. (3), the reduction in magnification helps to offset the reduced light collecting ability of lower NA objectives.

\section{Port Selection}

Although some manufacturers often do not make these data available, optical components and their coatings have dispersion curves, which plot \% transmission versus wavelength. As microscopes do not have $100 \%$ transmission at all wavelengths, losses will take place at certain wavelengths. ${ }^{6}$ Microscopes are built for optimal transmission in the green, because this is precisely what biologists want. Despite the fact that modern antireflection coatings are quite advanced (these vary among manufacturers and their composition is generally viewed as proprietary), losses take place, especially in the UV and violet. At $400 \mathrm{~nm}$, about $5 \%$ of the illumination intensity is lost at each interface. With, say, 20 optical interfaces at the top port of a typical inverted microscope, about one-half of the light is lost. To minimize the impact of these losses, an inverted microscope with a bottom port may be used. In this configuration, fewer optical interfaces are found between the emission filter and the detector.

\section{Filter Selection to Minimize Shot Noise}

Another strategy to preserve wanted light is to optimize the interference filters used in the microscope. This approach seeks to maximize the number of sample photons collected to minimize shot noise, which is a fundamental property of light. The relative contribution of shot noise to data will always decrease as the intensity increases (although other sources of noise could increase). Stern et al. (1989) have shown theoretically and experimentally that the shot noise in ratiometric indo-1 calcium studies is substantially reduced using custom broad bandpass interference filters. In outfitting microscopes with new filters, however, it is important to remember that both the wavelengths transmitted and the wavelength-dependence of reflectance are important considerations. Although this does not necessarily mean that calcium concentrations can be measured with greater precision, improved calcium signal/noise ratios have been observed (Stern et al., 1989). As the total number of photons is increased, imaging experiments are improved because the signal is further removed from the read noise floor.

\section{Minimizing Illumination Time}

In some situations, it may be important to minimize illumination time. For example, small molecules $\left(D \sim 10^{-5} \mathrm{~cm}^{2} / \mathrm{s}\right.$.) diffuse distances larger than the Rayleigh resolution criterion in time periods much less than the shutter speeds used in conventional imaging. By comparing Einstein's diffusion equation and Rayleigh's criterion, the rms displacement of a small molecule equals the Rayleigh criterion at about $100 \mu \mathrm{s}$. To eliminate the effect of diffusional blurring of fluorescence, flash excitation can be used. In this approach a flashlamp provides a pulse of excitation light for each frame. Figure 6 illustrates the Perkin-Elmer FX-4400

\footnotetext{
${ }^{6}$ www.olympusmicro.com/primer/anatomy/imagebrightness.html.
}

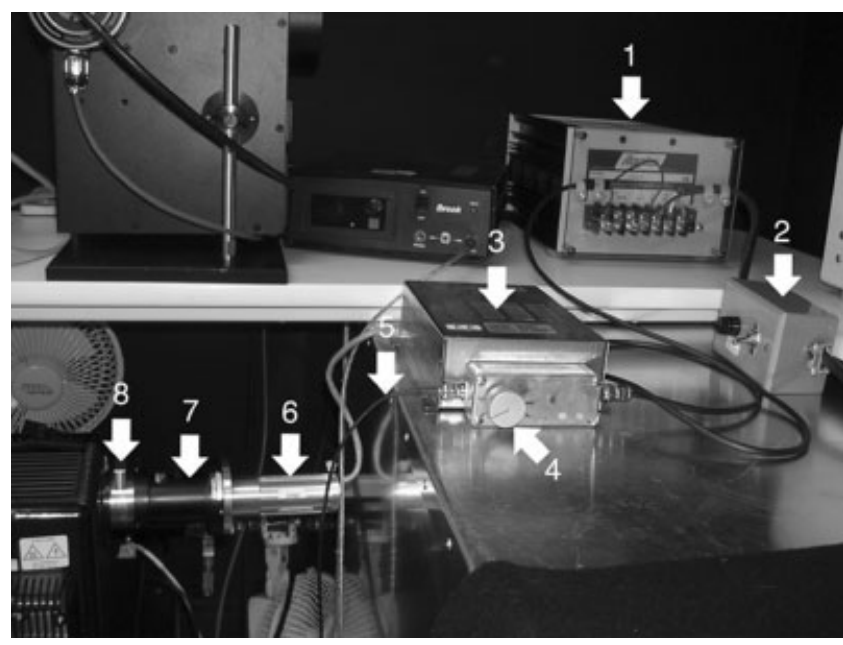

Fig. 6. Flashlamp illumination device for high speed fluorescence imaging. The components are labeled as follows: (1) $24-\mathrm{V}$ power supply, (2) on-off switch with fuse, (3) PS-4400 power supply, (4) voltage regulator for adjusting lamp brightness, (5) TTL input from camera, (6) FX-4400 bulb and FYD-4400 apparatus (Perkin-Elmer Optoelectronics) held in an adaptor for microscopes, (7) water filter for attenuation of UV and IR, (8) flange and slider holder for the Nikon white light TIRF apparatus.

flashlamp adapted for microscope imaging. This lamp provides an output of $1 \mathrm{~J}$ in $6 \mu \mathrm{s}$, which saturates dye molecules with light to maximize emission in a short time period. By driving the probe using brief, but intense, light pulses, the emission signal is high enough to avoid signal-to-noise issues. This collimated light is passed through a water filter to attenuate UV and IR components $\left(\sim 10^{9}-10^{10}\right.$-fold $)$ relative to visible wavelengths. The light can be further filtered using KG-1 and interference filters prior to entering the epifluorescence microscope. Figure 7A shows a raw image of indo-1 emission at $400 \mathrm{~nm}$ for a neutrophil following ingestion of opsonized zymosan taken with a $6 \mu$ s exposure of the FX-4400 lamp. For comparison, a similar image acquired with a standard mercury lamp is shown in Figure 7C. Although exposed for a $10^{4}$-fold shorter period of time, the flash excitation provides a suitable image of intracellular calcium indicator indo-1 in these stimulated cells after anisotropic diffusion filtering (Fig. 7B) (see also Anisotropic Diffusion, below). This particular micrograph was collected with an Andor iXon EMCCD camera because of the cells' robust calcium response under these conditions. However, for extremely low light levels an ICCD camera provides improved detection capability (Choosing a Detector, below). In addition, it should be possible to combine flash excitation with other microscope illumination modes, such as TIRF, to further dissect cell signaling pathways. In this way it is possible to remove the blurring effects of diffusion and flow of the calcium-indo-1 complex on the micrographs.

\section{CELLS, LABELS, AND EXPERIMENTAL DESIGN}

\section{Fixation and Background Noise Reduction}

Although the study of live cell dynamics has increased in recent years (Goldman and Spector, 2005), 

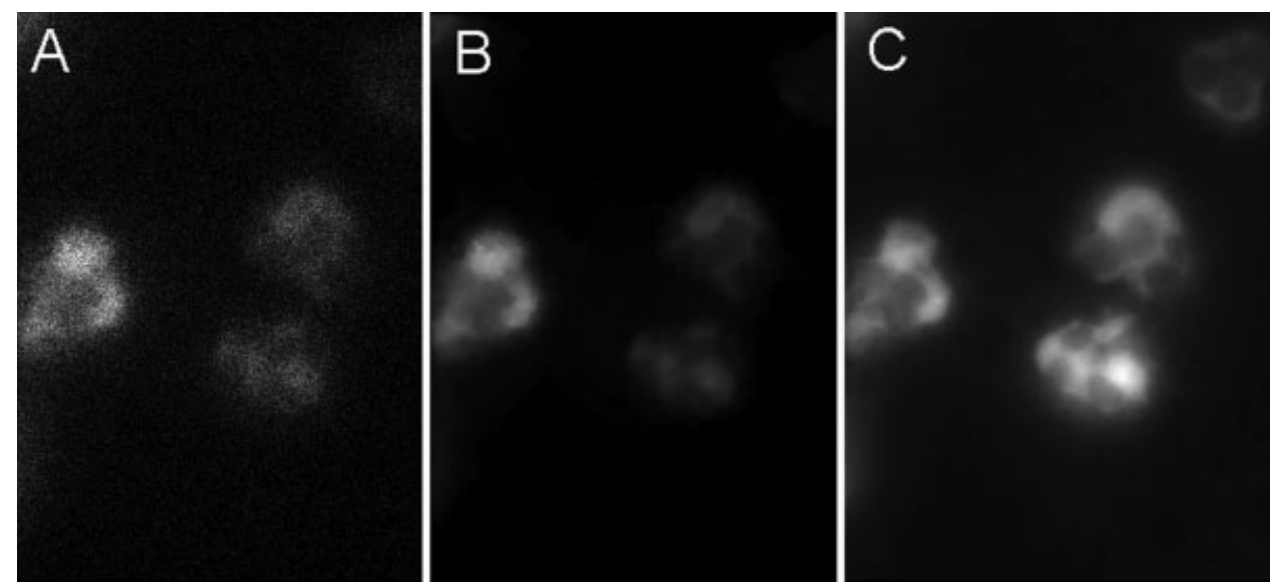

Fig. 7. Examples of images collected with flashlamp and conventional mercury illumination. Neutrophils were labeled with indo-1 followed by exposure to serum-opsonized zymosan. A: Single shot image of $6 \mu$ s duration using the apparatus shown in Figure 6. Indo-1 fluorescence is observed in these cells, especially in the vicinity of zymosan particles. B: The image of panel A after anisotropic diffusion fil-

methods to chemically fix or preserve cells and tissues will continue to play a major role in biology and medicine. The chemicals used most frequently in fixation are aldehydes, such as paraformaldehyde and glutaraldehyde. In typical protocols, samples are fixed for 15 min. to an hour, depending upon a sample's thickness. During this period of time, however, cell structures might undergo significant changes as cells die during fixation. For example, microtubules disassemble during fixation. To preserve such structures, it is necessary to rapidly fix a sample. More hydrophobic fixatives, such as dithio-bis(succinimidyl propionate) (DSP), cross membranes rapidly to preserve delicate intracellular structures and are generally used in combination with conventional fixatives (Safiejko-Mroczka and Bell, 1996). Thus, if fixation is being used, careful consideration of the methods is necessary.

One undesirable consequence of fixation is sample autofluorescence. Aldehyde-fixed samples autofluoresce due to Schiff's base formation (Clancy and Cauller, 1998). Several methods have been developed to minimize the fixation-autofluorescence problem. One way to manage this is to react the sample with $\mathrm{NaBH}_{4}$ to reduce the Schiff base (Baschong et al., 2001; Clancy and Cauller, 1998). Additional chemical means have been utilized to reduce background fluorescence. Exposure to ammonia-ethanol solutions is believed to improve the extraction of autofluorescent components whereas Sudan Black B is believed to mask these components (Baschong et al., 2001). It is also possible to minimize fixative-induced autofluorescence by taking advantage of photobleaching. Fixed samples are placed in a light box then exposed to the wavelengths of interest for extended periods of time to photobleach the autofluorescent molecules (Neumann and Gabel, 2002). The samples are later labeled with fluorescent antibodies to yield a dramatically improved signal-to-noise ratio. Finally, as the backgound autofluorescence has a short lifetime (Steinkamp et al., 1999), it is possible to use time-gated imaging to remove autofluorescence from the image (see Time-Gated Imaging). tration. C: A time-averaged indo-1 signal of the same cells acquired for $100 \mathrm{~ms}$ using a conventional Nikon HBO $100-\mathrm{W}$ lamp. Note that the cell on the left hand side is similar in panels $\mathrm{A}$ and $\mathrm{C}$. The two cells on the right hand side may be dimmer in panels $\mathrm{A}$ and $\mathrm{B}$ due to time-dependent changes in calcium levels.

\section{Cells and Protocols}

For live cell experiments, the cells under study can have profound effects upon their labeling. Thorough studies of cells and dyes must be performed. Cell properties may affect the stability of fluorescent probes. For example, a highly active glutathione cycle can reduce oxidant accumulation and the photobleaching of dyes in cells. By the same token, cells that produce large quantities of oxidative molecules can contribute to the photochemical decomposition of probes (Hafeman et al., 1984).

Several common problems are encountered in cell labeling. For dyes used to measure concentrations, such as calcium concentrations, it is possible to excessively label cells. Overloaded cells can buffer biological responses; thus, the label causes the signal to disappear. Another common problem is contamination of stock dye solutions with water. This can happen in many different ways, including condensation of water on the inside surface of dye vials. Water contamination leads to labeling artifacts, especially clumps of intracellular fluorescence (Kong et al., 1999; Lee et al., 1996; Lui et al., 1997). To minimize contamination, fluorescent dyes should be dissolved in dry DMSO (Lee et al., 1996). Dye solvents should be the highest quality dry solvent available and stored under desiccation. Dye leakage from labeled cells is another common problem that leads to rapid degradation in the signal-to-noise ratio. When dyes are delivered intracellularly as diacetate derivatives, these residues are cleaved to yield charged groups that trap them within a cell. However, these molecules may exit cells via anion transporters. The addition of anion transport inhibitors to the extracellular medium may help with some cells, but may severely damage other cells. Indeed, in our hands the morphological damage was so severe that this strategy was discarded. In some cases, derivatives with additional charged groups reduce the leakage rate; for example, the fluo-3 derivative LOJO (Teflabs) leaks from cells much more slowly than fluo-3. Alternatively, the dye can be chemically linked to a large molecule such as dextran then microinjected into a cell. It is 


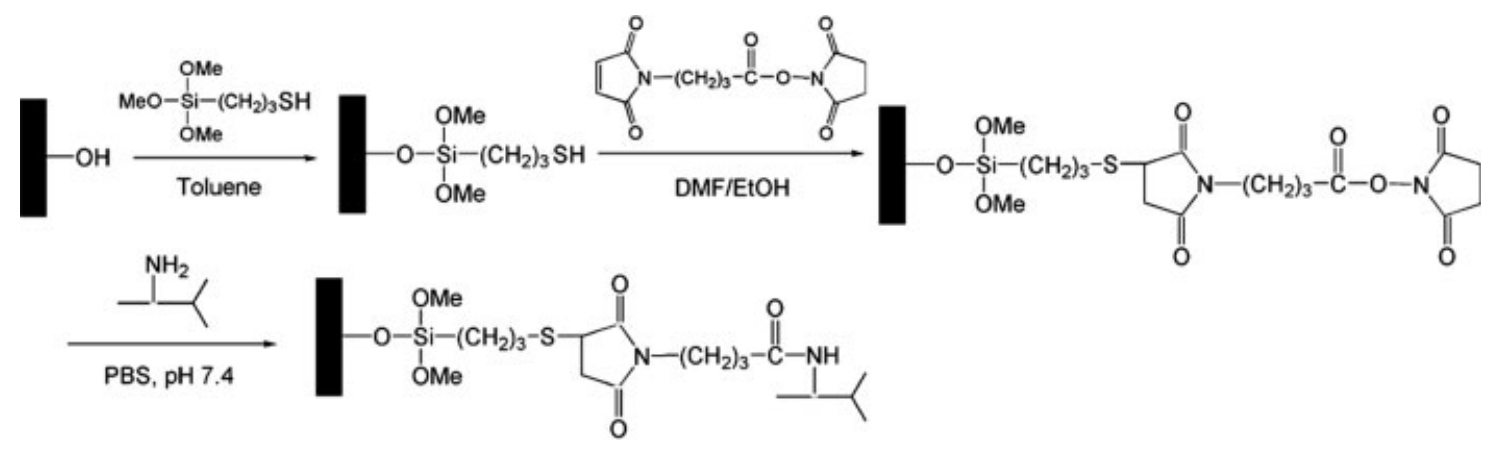

Fig. 8. Chemical route to modify glass cover slips. This figure illustrates the chemical reactions and solvents necessary to modify glass cover slips with Ab molecules.

often possible to avoid these problems if they are anticipated in advance.

Another factor to be considered in protocol development is the cover slip. Although cover-slips may be advertised as "precleaned" this does not mean that they should not be cleaned prior to experiments. In general, microscope cover-slips should be flamed and allowed to cool prior to use to remove any adsorbed materials. For example, neutrophils will polarize far better on a properly flamed cover slip. Cover-slips may also be cleaned with organic solvents. Biological molecules are frequently adsorbed to the surface of coverslips. These include materials such as collagen, poly-Llysine, BSA, and Abs. Although some materials such as poly-L-lysine and Cell-Tak (BD Biosciences) bind avidly to glass, others may not. The simple adsorption of ligands or Abs to glass cover-slips is inelegant at best. Using the straight-forward synthetic route illustrated in Figure 8, it is possible to attach a large variety of haptens, Abs, and other factors to cleaned glass coverslips. In this fashion, any doubt that the ligand or factor is displayed by the cover slip is removed. This approach is particularly useful in TIRF microscopy, as cells must form tight associations with the cover slip for TIRF excitation.

On the other hand, nonspecific adsorption of fluorescently labeled biomolecules to cover slip surfaces reduces the signal-to-noise ratio and degrades image quality. This may be observed during cell labeling with fluorescent $\mathrm{Ab}$ molecules. To minimize nonspecific adsorption, fluorescent Abs should be centrifuged to remove aggregates prior to staining. Moreover, nonspecific binding sites should be blocked; this is often accomplished by incubating samples with BSA. A commercial product called Image-iT (Invitrogen, Molecular Probes, Eugene, OR) has been introduced to block nonspecific staining using fluorescent labels.

\section{Organic Molecules as Labels}

Fluorescent reporters are either intrinsic or extrinsic in nature. Intrinsic fluorescent reporters include molecules such as tyrosine, tryptophan, NADH, and FAD. The autofluorescence of NADH and FAD are particularly interesting, because they act as intrinsic beacons of cell metabolism. Intrinsic reporters can also be genetically engineered into cells or animals, using probes such as green fluorescent protein (GFP) (see the following section). Extrinsic reporters are a large group of or- ganic dyes, such as fluorescein, or a small group of inorganic particles that fluoresce. By far, small organic molecules are the principle means used for the fluorescent labeling of molecules, cells, and tissues. Because this is likely to be the case for many years to come, it is important to be familiar with this broad class of compounds. One excellent source of information concerning these traditional labels is the "Molecular Probes Handbook" (Invitrogen, Molecular Probes, Eugene, OR).

As a rule, extrinsic fluorescent labels are designed to be excited near the emission peaks of mercury lamps $(313,334,365,406,435,546,578 \mathrm{~nm})$ used in microscopy. Fluorescent probes whose absorption does not correspond to these peaks are less well excited than probes absorbing light at these wavelengths. For example, more relevant light is delivered to AMCA, NBD, and rhodamine than fluorescein in a microscope equipped with a mercury lamp because there is no mercury line near $490 \mathrm{~nm}$. An argon ion laser's line at $488 \mathrm{~nm}$ is a much better excitation source than a mercury lamp. On the other hand, a xenon lamp has a very broad emission spectrum and thereby excites many molecules equally well (this is why xenon lamps, not mercury lamps, are used to collect excitation spectra). However, xenon lamps are not as bright as mercury lamps. When a fluorochrome's excitation maximum does not correspond to a lamp's emission peaks, it is best to use excitation filters with a wide band pass. These features should be considered in designing the most appropriate experiments.

Another fundamental physical property of fluorescent labels is that their emission properties depend upon their chemical structure and environment. In general, the larger the fluorescent label (or more specifically, its pi electron cloud), the longer the emission wavelength. During excitation, the dipole moment of a dye is increased. This property can be exploited in fluorescence microscopy experiments. As mentioned in Fluorescence, when a dye is in a hydrophobic environment such as a membrane, it requires more excitation energy because of the excited state's polarity. This can be used to characterize molecules in different environments or to identify a molecule's location. For example, this strategy has been used to identify lipid rafts in cell membranes (Kindzelskii et al., 2004).

A great variety of fluorescent probes are commercially available. One of the most common probes is fluorescein isothiocyanate (FITC). For several reasons, nei- 
ther the fluorescein dye nor the isothiocyanate linker is the best choice. Although $480 \mathrm{~nm}$ excitation is not optimal for a mercury lamp, there are probes other than FITC that have better physical and chemical properties; these include BODIPY FL, Alexa fluor-488, and Oregon Green. These probes are characterized by better stability, higher quantum yields, and/or reduced susceptibility to fluorescence quenching. Two routes to reduce dye photobleaching are: (1) minimize the probability of triplet state formation and (2) reduce chemical sensitivity to oxidants. Recently, polyfluorination of a cyanine dye has been shown to increase stability and improve fluorescence characteristics (Renikuntla et al., 2004). Furthermore, fluorophores designed for conventional imaging applications may not be the best for specialized applications such as two-photon excitation, as the absorption cross-sections for signal and two-photon imaging many not be equivalent. Moreover, dyes used in single photon excitation may be photochemically destroyed at a much higher rate during two-photon excitation (Dittrich and Schwille, 2001). Schafer-Hales et al. (2005) have reported that dyes incorporating fluorine are superior in two-photon microscopy. As mentioned in Cells and Protocols, dye leakage can be reduced using more highly charged derivatives, such as LOJO. Hence, a great variety of fluorescent labels are now available that can, in some cases, be tailored to specific experimental conditions or to relieve certain experimental difficulties.

\section{Intrinsic Labeling by Genetic Means}

The advent of intrinsic genetic labeling protocols using GFP and related molecules has provided an important new set of minimally invasive fluorescent tools in cell and tissue biology (Chudakov et al., 2005). GFP is a barrel-shaped $28 \mathrm{kDa}$ protein originating from jellyfish. Its fluorescent moiety is formed by three cyclized and oxidized amino acids at the barrel's center. Single point mutations were introduced into the GFP gene to improve its biological and physical characteristics. For example, its expression was enhanced by changing codon usage from jellyfish to vertebrate and by improving protein folding in higher eukaryotes. The fluorescence properties were improved using point mutations to alter the local dielectric constant near its fluorescent moiety (these mutations caused a solvent effect at the active site that changed the energy level spacing). Collectively, this altered protein is known as enhanced GFP (EGFP). By further altering the polarity near the fluorescent moiety, blue, cyan, and yellow fluorescent proteins have been engineered (Shaner et al., 2005). Other organisms have been used to identify new FPs to extend the accessible FP spectrum deeper into the red, such as DsRed, which absorbs at $558 \mathrm{~nm}$ and emits at $583 \mathrm{~nm}$. Often, FPs are used in the form of chimeric proteins to specifically label a protein of interest. FPs have also been transduced into many animal and plant model systems for scientific as well as commercial purposes (Stewart, 2006).

FPs have found numerous applications in biomedical research. One chief application of FPs is in protein localization and trafficking. FPs are also used in gene reporter studies of target gene up/down regulation. In combination with FRET microscopy, FP can be used to study protein-protein interactions and the intracellular evolution of these interactions over time. However, in some instances formation of a chimeric protein containing a GFP and target protein will disrupt the latter's normal functions. As some FPs may dimerize, this potential artifact should be checked in experimental designs.

In addition to protein localization and interactions, FPs are also used as chemical sensors of $\mathrm{Ca}^{2+}, \mathrm{pH}$, and $\mathrm{Cl}^{-}$(Chudakov et al., 2005; Rogers et al., 2005). GFP fluorescence is intrinsically $\mathrm{pH}$-sensitive. Mutations of this molecule have led to the development of FPs with improved $\mathrm{pH}$-dependent emission spectra. An important class of sensors reports calcium levels. For example, one class known as cameleons (Miyawaki et al., 1997) are constructed by the linear fusion of sequences encoding blue-emitting FP, calmodulin, a calmodulinbinding protein, and a green FP. In the presence of calcium, the calmodulin and calmodulin-binding proteins interact to shorten the probes length thereby increasing the energy transfer between the two FPs. Several additional types of detectors have also been generated (Griesbeck, 2004). Furthermore, transgenic mice expressing calcium reporter proteins have been constructed (Hasan et al., 2004; Pologruto et al., 2004). However, the performance of these calcium sensors is poor ( $\sim 3$ - to 5-fold change in contrast) in comparison to fluorescent dyes that undergo much larger changes in emission.

GFP and related molecules are also important in whole-animal studies. Using GFP-tagged target or effector cells in combination with stereomicroscopy, it is possible to study the trafficking of these cells in living animals. Using $E$. coli that express GFP, the process of infection has been visualized (Zhao et al., 2001). Similarly, it is possible to follow the staging and metastasis of cancer cells in vivo (Yang et al., 2002). In addition, GFP-tagged leukocytes have been used to follow their in vivo trafficking (Grayson et al., 2003; Sonoda et al., 2005; Srivastava et al., 2005). By extension, it should be possible to follow leukocyte trafficking in tumor-bearing animals. Further developments in the use of GFP and related molecules should continue to illuminate in vivo immunological processes.

\section{Nanocrystal Labeling}

In addition to the conventional labeling protocols described above, there are several emerging nanoparticle labels that possess certain advantages as fluorescent probes. The best known of these labels are quantum dots, which constitute an entirely new class of fluorescent labels (Alivisatos et al., 2005; Gao et al., 2005; Jaiswal and Simon, 2004; Michalet et al., 2005; Smith et al., 2004a). These labels are now commercially available. In contrast to the organic molecules mentioned above, quantum dots are inorganic semiconductor nanocrystals composed of CdSe, CdS, CdTe, InP, or InAs between 1 and $10 \mathrm{~nm}$ in size. Generally, quantum dots are coated for biocompatibility and to provide chemical groups for the attachment of peptides, proteins, nucleic acids, etc. and to reduce toxicity. Quantum dots emit fluorescence from 400 to $2,000 \mathrm{~nm}$. Just as the emission maxima of organic fluorochromes depend upon the molecule's size, the emission wavelengths of quantum dots depends upon their size with smaller dots emitting blue light and larger dots emit- 
ting infrared light. Although the emission spectra are narrow, their excitation spectra are extremely broad. Quantum dots exhibit long fluorescence lifetimes ( 20-50 ns), which should make time-gated experiments very useful. Their comparatively large two-photon absorption cross sections make them appropriate for two-photon imaging experiments. Quantum dots are very stable: photobleaching is negligible. Because of their electron density, quantum dots can be visualized using electron microscopy. Hence, quantum dots have properties that make them useful in several labeling applications (Alivisatos et al., 2005; Liu et al., 2005).

One application of quantum dots is multi-color labeling; their broad absorption characteristics allow one excitation wavelength to excite several quantum dots of different colors while their narrow emission bands allow multiple labels to be easily distinguished. Exceptional brightness and minimal photobleaching make quantum dots an excellent choice for studying molecules in cells expressed at very low copy number. Single molecule tracking in single cells is now possible with this tool. Similarly, their stability makes quantum dots essential for long time scale experiments. Quantum dots have been used in cell trafficking studies in vivo (Gao et al., 2005), including cell trafficking to tumors and lymph nodes. Although the long lifetime of quantum dots has not yet been exploited in biological applications, it is clear that very low noise/high contrast images containing quantum dots could be generated by time-gated experiments wherein autofluorescence is gated out of the image and illumination noise is removed by averaging many frames. Time-gated noise reduction and near infrared quantum dots (greater tissue penetration of excitation light) will be particularly useful in whole animal and tissue studies. Furthermore, cell labeling strategies employing multiple quantum dots with differing spectroscopic signatures will be useful in highly complex trafficking studies of multiple cell types. However, despite these advantages, quantum dots are relatively expensive, and they will not soon replace the use of organic dyes.

Quantum dots are also useful as donors in FRET studies. However, their use as acceptors is not possible because of their broad excitation spectra. Similarly, experiments requiring the selective excitation of specific labels are not appropriate. Quantum dots cannot be recommended for quantitative studies. First, because of transitions between singlet and triplet states, quantum dots can exhibit blinking fluorescence, a random switching between fluorescent and nonfluorescent states. A second issue in quantitative studies is photobrightening, which is an increase in fluorescence intensity upon excitation. However, this is an advantage for detecting and locating molecules.

In addition to quantum dots, two additional nanocrystalline materials are emerging as potential fluorescent labels. Nanodiamond particles with nitrogen-containing point defects absorb at $560 \mathrm{~nm}$ and emit fluorescence at $700 \mathrm{~nm}$ with a lifetime of $\sim 11 \mathrm{~ns}$. These particles experience negligible photobleaching over periods of hours and can withstand extremely high light levels ( $\mathrm{Yu}$ et al., 2005). Other nanocrystals made of $\mathrm{Gd}_{2} \mathrm{O}_{3}$ or $\mathrm{Y}_{2} \mathrm{O}_{3}$ containing small amounts of $\mathrm{Eu}$ or $\mathrm{Tb}$ exhibit fluorescence emission with a lifetime of about one ms (Dosev et al., 2005; Nichkova et al., 2006). Although these particles have very little photobleaching and sharp emission spectra, their long lifetimes make them less useful in certain applications. However, they have been shown to be useful in fluorescence immunoassays (Cummins et al., 2006).

\section{Image Ratioing Microscopy}

Image ratioing microscopy began as a method to correct for variations in cell thickness, for example, by labeling living cells with fluorescent actin and a nonspecific label distributed throughout the cytoplasm (Taylor et al., 1980). Image ratioing microscopy is now used to detect many chemical changes within cells, such as the $\mathrm{Ca}^{+2}, \mathrm{Na}^{+}$, and $\mathrm{pH}$ changes. Tsien and coworkers have developed a variety of compounds that detect ions by ratioing images collected using different excitation wavelengths or emission wavelengths (Minta et al., 1989). These dyes exhibit a shift in the fluorescence excitation or emission spectrum in response to ion binding. For example, when the calcium probe indo- 1 binds to calcium, its emission at 400 $\mathrm{nm}$ brightens whereas its emission at $480 \mathrm{~nm}$ dims. The effective dynamic range is increased by ratioing, which can be used to better calculate calcium concentrations. Furthermore, it is possible to obtain dyes with $K_{\mathrm{d}}$ s varying over several orders of magnitude, which permits more specialized experimental designs. One of the advantages of ratioing is that it corrects for variations in the amount of dye in the optical path, which could arise due to dye compartmentalization or dye loss due to photobleaching or leakage. Image ratioing also compensates for variations in the intensity of the exciting light, provided that the two images or readings are collected simultaneously. A disadvantage of ratioing is that noise increases; for example, the shot noise of a nonratiometric image is $\sqrt{ } \mathrm{N}$ whereas that of a ratiometric image is $\sqrt{2 N} .^{7}$ Indeed, because of the propagation of error, noise will always increase as the number of mathematical manipulations increase. Another disadvantage of image ratioing is that it is often slow. For example, if ratioing involves collecting and downloading two images and moving a shutter wheel between two different positions, this will require at least several tenths of a second, which is very slow compared to signaling kinetics and ion channel activity. Recently, dual emission wavelength imaging systems have become commercially available (Table 2), which allow multiple emission wavelengths to be simultaneously photographed. For emission wavelength ratioing microscopy, these dual imaging systems greatly increase the speed of data collection and allow simultaneous evaluation of live cell events.

\section{PHOTOBLEACHING Nature of Photobleaching}

Illumination is a requirement in obtaining fluorescence emission. When using a conventional lamp, the illumination is always adjusted for Köhler illumination and re-adjusted when the objective is changed. The optimal illumination level, however, may not be

\footnotetext{
${ }^{7}$ It is easy to see that this is not $2 \sqrt{ } \mathrm{N}$ because the noise is random and occasionally cancels out.
} 
obvious. The fluorescence emission increases as the level of illumination increases until a saturation point is reached. When illumination is increased beyond saturation, the total number of photons reaching the detector will increase, but this is because of the fact that background scattering continues to increase with excitation intensity; the fluorescence photons remain constant while the signal-to-noise ratio decreases (Mathies et al., 1990). As a rule, conventional mercury lamps are unable to saturate a labeled cell; pulsed laser and flashlamps can reach illumination levels needed to saturate a fluorescent dyes.

Photobleaching is the chemical destruction of fluorescent molecules as a result of excitation, which can occur at timescales of $\mathrm{ms}$ to minutes. Photobleaching is an incompletely understood heterogeneous physical and chemical process that may vary among dyes (Koizumi and Usui, 1972; Song et al., 1995, 1996). In some cases, photobleaching has been found to involve the participation of triplet dye states. Both oxygen-dependent and oxygen-independent mechanisms contribute to photobleaching. The mechanism of photobleaching may vary from one dye to another. The fluorochrome's concentration may also influence the mechanism of photobleaching (Song et al., 1997): at lower concentrations the dye may interact with oxidizing or reducing agents but at higher concentrations dye molecules may interact with themselves (e.g., singlet-triplet interactions). Photobleaching may be sensitive to conditions such as temperature and polarity of the environment (Eggeling et al., 2005). As some cells possess a robust glutathione cycle, a diminished rate of intracellular photobleaching would be expected for these cells, in comparison to cells having a less tightly controlled redox environment. In contrast, cells producing superoxide anions have accelerated photobleaching (Hafeman et al., 1984). Photobleaching may differ for molecules in solution versus molecules attached to cells. For both dyes and fluorescent proteins such as GFP or light harvesting complexes, singlet oxygen and superoxide anions are key molecules mediating photobleaching (Greenbaum et al., 2000; Song et al., 1995, 1996). Photobleaching depends upon exposure time. When a sample does not absorb light (for example, when the light is shuttered or the dye does not absorb light), a sample is not photobleached. Finally, higher illumination levels may accelerate photobleaching beyond levels extrapolated from low intensity illumination levels (Bernas et al., 2004; Patterson and Piston, 2000).

\section{Photobleaching Management}

Several means of controlling photobleaching are available to the experimentalist and the optimal strategy depends upon both the sample and the type of fluorescence microscopy. One strategy to reduce photobleaching is to reduce the illumination level by inserting neutral density filters into the light path or reducing lamp output. Another simple approach to diminish photobleaching is to use a less sensitive fluorophore. For example, polyfluorinated dyes and Alexa dyes are more stable than conventional dyes (PanchukVoloshina et al., 1999; Renikuntla et al., 2004). The time required to download images from detectors to computers can, in some cases, become a significant fraction of the camera's duty cycle. This is especially

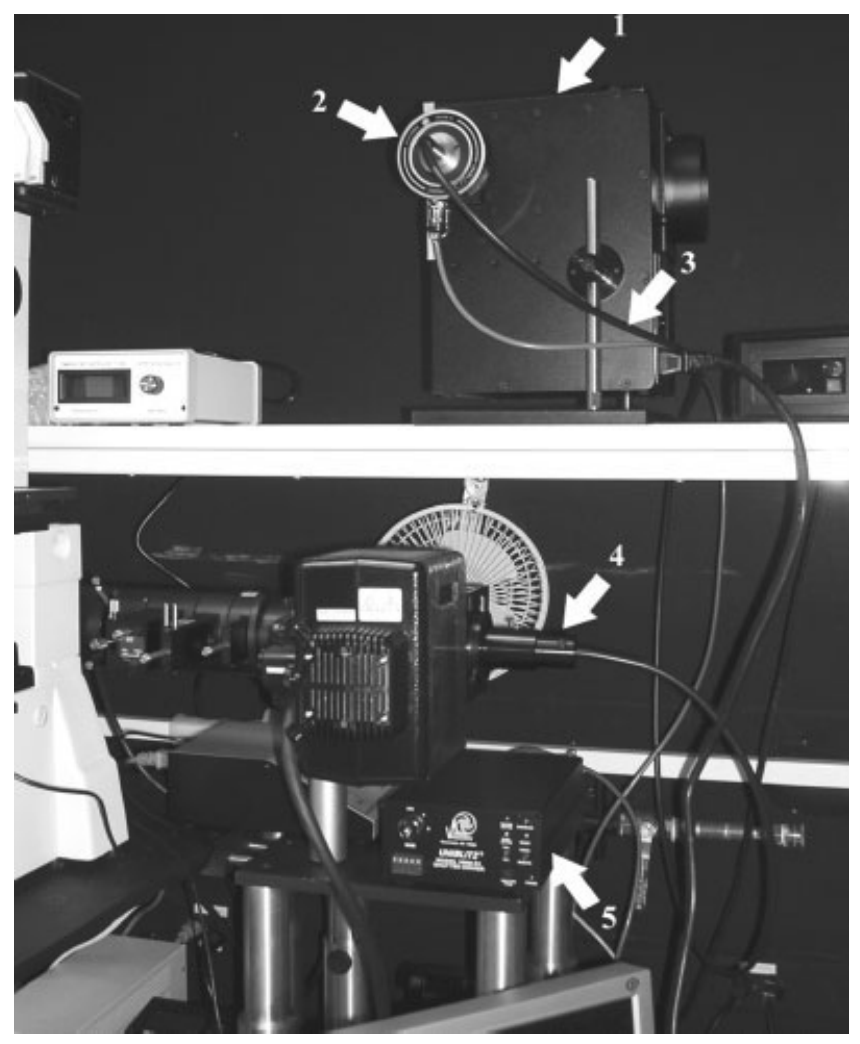

Fig. 9. Apparatus for rapid shuttering of a xenon light source. The components are numbered as follows: (1) Sutter lamp, (2) Uniblitz high speed laser shutter, (3) optical pipe, (4) microscope adapter, and (5) Uniblitz shutter control system. This system can be rapidly opened and closed to minimize sample photobleaching.

true at higher shutter rates or while downloading images at slower speeds to reduce readout noise. To minimize photobleaching, the excitation light can be shuttered. Although electro-optic shuttering can be employed, relatively low cost mechanical shuttering can be used at rates of up to $300-400 \mathrm{~Hz}$. In this case, laser shutters can be used with lasers or with light guides to provide illumination (Fig. 9) and electronically synchronized with the camera system.

Another approach to manage photobleaching is to inhibit photochemical decomposition. For fixed samples, these approaches should be routinely employed. However, for live cells careful consideration is required as these chemical means of minimizing photobleaching may also affect the pathways the experiment is intended to illuminate. Early studies demonstrated that the fluorescence of fixed cells could be better preserved using p-phenylenediamine (Johnson et al., 1981, 1982). This and other antiphotobleaching compounds such as $n$-propylgallate, ascorbic acid, and dithionite are electron donors for triplet fluorophores and thereby lower the concentration of the reactive triplet during photoexcitation (Hafeman et al., 1984). Ono et al. (2001) compared a variety of available kits to reduce the photobleaching of fixed samples. These kits vary in their composition and properties. For example, some kits greatly inhibit photobleaching but, in the process, reduce sample brightness. 
A second chemical strategy to reduce photobleaching is to remove oxygen (Bernas et al., 2004), which of course may have untoward effects on living cells. Oxygen can be removed by bubbling argon through the solution. To insure that the oxygen concentration is low, glucose and glucose oxidase can be included with the sample. Oxygen removal can be confirmed using oxygen-sensing probes (e.g., $\mathrm{Ru}(\text { phen })_{3}{ }^{+2}$ ). Another strategy to reduce the concentration of superoxide anions in a sample, and their potential contribution to photobleaching, is to include superoxide dismutase. In cases where dissolved oxygen is removed, it is important to insure that a tight seal is used to prevent oxygen entry into the sample. Anoxia is observed in some biological settings and can be designed as an important aspect of the protocol. For example, anoxia may be associated with inflammatory sites, ischemia-reperfusion events, and certain tumors; hence anoxia can be used to reduce photobleaching and improve the experiment's physiologic relevance.

Another factor that leads to the fading of fluorescence as well as cellular damage is the constant infrared light produced by a typical lamp, which heats a sample. Infrared blocking filters should be in the excitation apparatus of the microscope. If necessary, infrared filters can also be inserted into the fluorescence filter holders of a microscope. This not only minimizes cellular artifacts and fading, but also helps to prolong the lifetime of interference filters. As CCD detectors are particularly sensitive to infrared light, even small amounts of infrared light cause background haze that degrades image contrast. When using mercury lamps with outputs over $100 \mathrm{~W}$, for safety reasons, it is important to use air filters to remove the ozone generated by the lamp and water filters to remove infrared light from the output.

\section{Computational Compensation for Photobleaching}

If adjustments to the label, illumination, and media conditions are not sufficient to control photobleaching, it is possible to manipulate the resulting images using software tools to minimize the effects of photobleaching. The AndorIQ (Andor Technology) software package has a photobleaching compensation program. For example, as the dynamics of a fluorescent molecule is studied over time, it progressively fades. To correct for photobleaching, these data can be compared to an unchanging region of the sample. Alternatively, the fluorescence of the entire image can be used for normalization. However, this assumes that the total intensity of the sample is constant; if this is not true, an internal unchanging control, such as a fluorescent bead, is required.

In addition to this computational correction for photobleaching, it is also possible to "rescue" information in a degraded image using image filtration approaches, such as anisotropic diffusion and wavelet processing. These methods will be discussed below (Processing).

\section{CAMERAS}

A principal source of noise in fluorescence microscopy is the detector. Most imaging detectors (cameras in contrast to photomultiplier tubes) in use are now digital CCD cameras. Digital cameras have many advantages over prior video technology, which we will not present here. The CCD detector, per se, is an analog optical device generating output voltages. These voltages undergo amplification and analog-to-digital conversion on-board the chip, in contrast to video technology wherein the $\mathrm{A} / \mathrm{D}$ conversion takes place inside a computer. Lower noise levels are associated with digital cameras. Nonetheless, CCD cameras have multiple sources of noise, which will be outlined below.

\section{CCD Noise}

Photon or shot noise is one type of noise encountered during light detection. It is because of the stochastic nature of photon arrival at the detector. The magnitude of shot noise is the square root of the total number of counts. As shot noise is a fundamental property of light, it cannot be eliminated from the CCD output. However, steps can be taken to minimize shot noise (Reducing Illumination Noise) and recent computational methods have been used to recognize and adjust for this source of noise in images under special circumstances (Uttenweiler et al., 2003).

Another important source of detector noise is dark noise. As its name implies, dark noise is independent of illumination. Dark noise arises from thermally generated electrons within the CCD chip, which are indistinguishable from photoelectrons. These spurious electrons are released during exposure of the chip and during the read-out phase, while the information is waiting to leave the chip. As the chip's temperature is decreased, the number of thermally generated electrons decreases. The dark noise decreases 10-fold for each $20^{\circ} \mathrm{C}$ reduction in chip temperature, although this effect becomes negligible at lower temperatures. This reduction in dark noise is an important reason why low light level detectors are cooled from $-20^{\circ} \mathrm{C}$ to $-100^{\circ} \mathrm{C}$. In some cases, dark current can take the form of "hot" pixels. Impurities in the silicon can cause hot pixels, which can also be reduced by cooling.

Noise is also generated after the light has been converted into photoelectrons. Read-out noise is generated during the conversion of photoelectrons in a CCD well into a digital value. For example, when charge is being transferred between wells during the read-out process, an electron might jump ahead too far, in the wrong direction, or not move at all. Read-out noise is also generated by the output amplifier and, to a lesser extent, on-chip electronics. The magnitude of the read-out noise depends upon the read-out speed, the chip's electronics, and temperature (Coates et al., 2004; Hynecek and Nishiwaki, 2003; Smith et al., 2004b). The importance of read-out noise in the final signal-to-noise ratio depends upon the type of detector being used, which will be discussed in the following section.

\section{Chip Architecture}

The architecture of the detector's chip can vary quite a bit. Both CCD and CMOS (complementary metal oxide semiconductor) chips are used in digital cameras (Magnan, 2003; Theuwissen and Roks, 2001). CCDs are used primarily for scientific imaging purposes, although CMOS cameras are used in ballistics and other applications requiring high frame rates. Although there may be some advantages in CMOS cameras, their development has lagged behind that of 
CCDs. (Although it should be possible to construct CMOS sensors with low readout noise, they are noisier and less sensitive than CCDs.) CCD cameras can be: full frame, frame transfer, or interline transfer detectors. The key difference in chip design is that in frame transfer and interline transfer architectures the photoelectrons are temporarily stored in sets of masked wells (either in the shape of a frame or a line) prior to being read out into a computer. Although masking wells makes the chip more expensive and may reduce the light detection area, it allows the slow read-out process to continue while the next frame is being exposed. Another variable in chip architecture is front-illuminated versus back-illuminated. Back-illuminated chips are "thinned" by removing much of the substrate the chip is printed on. These chips do not suffer losses due to surface channels, but they are also more fragile than front-illuminated chips. Back-illuminated chips have quantum efficiencies as high as 95\%, whereas typical efficiencies of front-illuminated chips are about $50 \%$. Hence, back-illuminated chips are preferable in low light level applications (Coates et al., 2004).

To detect low levels of fluorescence, the signal must be amplified with as little added noise as possible. The signal can be amplified either before the formation of photoelectrons (intensified CCDs; ICCDs) or after the creation of photoelectrons (electron-multiplying CCDs; EMCCDs). In ICCDs a multichannel plate (MCP) provides electron multiplication. During the light intensification phase, light strikes a photocathode to generate photoelectrons. An applied voltage accelerates the electrons across the MCP, which consists of bundles of small $(6-12 \mu \mathrm{m})$ capillary fibers. Electron collisions with the capillary's walls cause additional electrons to be released yielding 1,000-fold amplification. These electrons then strike a phosphorescent screen, which emits light detected by the CCD (see above description). As the phosphorescent screen has a quantum efficiency of $\sim 50 \%$, it is important that the $\lambda_{\max }$ of the phosphor matches that of the CCD chip. One advantage of ICCDs is their very fast gating $(\sim 1 \mathrm{~ns})$. Noise arises at several stages, including thermionic electron release, fluctuations in MCP gain and others. As amplification occurs before light strikes the CCD, much larger numbers of photoelectrons are measured thereby making read noise comparatively insignificant. Importantly, ICCDs can be damaged by exposure to excessive light levels and therefore bright-field microscopy must be avoided.

In contrast to ICCDs, EMCCDs are all solid-state devices that amplify the signal after light strikes the chip. Consequently, intensifier noise is not relevant to EMCCD cameras. In EMCCD chips, an electron multiplier is inserted after the shift register but before the output amplifier. As electrons are read-out, impact ionizations within the gain register cause additional electrons to be released; as this occurs repeatedly, substantial gains are possible. Dark noise is an important concern for EMCCD chips, as the gain register will multiply both the dark noise and the signal leading to poor signal/noise ratios at low light levels. Some manufacturers cool these chips to $-100^{\circ} \mathrm{C}$ to minimize noise. A key noise source for EMCCDs is read-out noise, specifically clocking-induced charge (or spurious noise), which dominates the noise of a deeply cooled EMCCD.
As electrons are moved among wells in chips outside the gain register, they can cause impact ionizations; this is the same physical process used for electron amplification in the gain register. Although this is usually small for CCDs and ICCDs, it is an important source of noise for EMCCDs, especially when the electron gain register is operating at high gain. At slow speeds, read-out noise might be only two or three electrons per pixel, whereas higher speeds could increase this to $>30$ electrons per pixel. Coates et al. (2004) provide a comprehensive discussion of EMCCD noise. An additional advantage of EMCCDs is improved spatial resolution (Denvir and Conroy, 2002). Most commercial EMCCDs have separate channels for electron multiplication and nonelectron multiplication modes, which allow for studies of weak fluorescence and bright-field illumination, respectively.

\section{Choosing a Detector}

There is no "best" detector to purchase for a laboratory. Each detector has its strengths and weaknesses. The specific application will determine which detector is most appropriate. It is best to test a variety of detectors in a particular application before purchasing one. In general, ICCDs are more sensitive at low light levels than EMCCD cameras (Dussault and Hoess, 2004). For exposure times of less than a few ms, the ICCD camera is superior to the EMCCD. On the other hand, at longer integration times the EMCCD camera provides the highest quality image. One manufacturer of ICCD cameras is Princeton Instruments. In my opinion, the intensified retiga of $\mathrm{Q}$ imaging is not reliable enough to be a useful instrument. Manufacturers of EMCCD cameras include Andor and Photometrics; in my experience these are both excellent devices.

Another factor that should be considered in the selection of a camera is the wavelength-dependent response of the detector. Detectors are generally built for specific applications, such as biomedical research; for this reason, detectors are generally built for optimal sensitivity in the green (for example, Gen3 intensifiers for ICCD cameras). In general, detectors should have greatest $\mathrm{QE}$ at the wavelength relevant to the particular experiment. For example, a fluorochrome or endogenous molecule such as NADH that emits in the violet would need a blue-enhanced detector. On the other hand, if the intent were to image the fluorescence of fullerenes, a camera capable of detecting fluorescence emission in the IR would be required.

Finally, some consideration to the pixel size and array size of the detector's chip should be given. Typical pixel sizes in these cameras range from $3.4 \times$ $3.4 \mu \mathrm{m}^{2}$ to $19 \times 19 \mu \mathrm{m}^{2}$. The optimum pixel size is influence by the objective(s) needed for the experiment, as suggested by Eq. (4). Although the spatial resolution increases as pixel size decreases, the full well capacity of the pixel decreases thereby reducing its maximal brightness and "bit depth." For example, a chip with $3.4 \times 3.4 \mu^{2}$ pixels may have a 10 bit gray scale whereas chips with $16 \times 16 \mu \mathrm{m}^{2}$ pixels often support images 16 bits deep. Large pixel sizes hold more electrons, but are preferably used with high magnification objectives. Additional lenses can be inserted to increase the microscope's magnification. Two common pixel array sizes of EMCCD and ICCD cameras are $512 \times$ 
512 (e.g., $16 \times 16 \mu^{2}$ for Andor's $512 \times 512$ iXon EMCCD camera) and 1,024 × 1,024 (e.g., $13 \times 13$ $\mu \mathrm{m}^{2}$ ). Not unexpectedly, the cost of a $1 \mathrm{~K} \times 1 \mathrm{~K}$ chip is substantially greater than that of a $512 \times 512$ chip. On the other hand, a $1 \mathrm{~K} \times 1 \mathrm{~K}$ chip provides four times as many data points, better resolution, and better resolution matching with common $100 \times$ objectives.

\section{PROCESSING}

In addition to playing indispensable roles in the image management and esthetic presentation, image processing also plays a key role in manipulating images to make their information more useful. There are many conventional applications of image processing. One application is background subtraction. Using segmentation routines, specific colors or gray levels can be extracted from an image. Quantification of image characteristics can also be performed using tools that measure image parameters. One can modify images by adjusting the gain and offset of a camera (brightness and contrast) to boost the intensity of a dark image or to bring out subtle features of particular interest. Images can be assigned a look-up-table (LUT) such that features of interest are readily apparent to viewers. Several reviews have discussed image processing methods in cell biology and immunology (Sabri et al., 1997); here we will focus on approaches most useful in managing image noise.

A number of computational filters are available for image processing to reduce the visual impact of noise. Although it is commonly believed that the signal-tonoise ratio must be $>1$ to extract information from an image, this is not true because noise is random and biological entities are not. Two filters used to extract weak images from data sets, which are generally considered to be preprocessing steps, are anisotropic diffusion and wavelet analysis, which will now be discussed. Although widely used in ultrasound and NMR, these software tools are hardly used in cell biology and immunology.

\section{Anisotropic Diffusion}

One example of a smoothing technique is Gaussian filtering (isotropic diffusion): each pixel is given a weighted average based upon its immediate neighbors. This reduces noise fluctuations, but degrades edges due to its blurring effects. Edge finding is very important in biology for tasks such as finding the edge of a tumor or a microtubule. In contrast to such smoothing methods, adaptive smoothing methods minimize noise while retaining edges. In this approach, smoothing depends upon the local properties of the image, which is a nonuniform or anisotropic method. Perona and Malik (1990) introduced anisotropic diffusion filtering of images. This approach uses a partial differential equation identical to the diffusion equation, except that a term is included to modify smoothing based upon the local rate of change in pixel intensity (a boundary). The basic assumption here is that a signal consists of oriented structures and that noise is uncorrelated and thus has no preferred orientation. This seems reasonable because edges are information carrying structures in images. Software to perform anisotropic diffusion is available commercially and through public sites (e.g., Image $\mathrm{J}$ ). Figures $7 \mathrm{~A}$ and $7 \mathrm{~B}$ illus- trate anisotropic diffusion filtering of a calcium image of neutrophil acquired with a $6 \mu$ s pulse of excitation light. For the bright cell on the left hand side of Figures $7 \mathrm{~B}$ and $7 \mathrm{C}$, the signal-to-noise ratio of the filtered image acquired for $6 \mu \mathrm{s}$ is comparable to that of a conventional mercury illumination source. Anisotropic diffusion filtering also performed well in preserving edges. In addition, anisotropic diffusion an also be used to filter three-dimensional data sets (Broser et al., 2004).

\section{Wavelet Analysis}

Wavelet analysis is a well-established tool in image analysis (Unser and Aldroubi, 1996), but rarely used in microscopy. A wavelet is a waveform of limited duration, which is, for example, a representation of a physical length in an image. These wavelets are scaled and translated to match the input signal. Just as a signal can be decomposed into a series of sine waves in Fourier analysis, a signal can be broken into a number of scaled and shifted versions of the original function. Wavelet analysis preserves edges and local information. Consequently, it is well-suited for the detection of image features and de-noising. Images can be decomposed into sets of images with different wavelet coefficients (Moss et al., 2005). As the spatial characteristics of noise differ from that of biological structures, the filtered output corresponding to specific sizes can be obtained, thus removing noise. This amounts to setting the wavelet coefficients associated with noise to zero and synthesizing the filtered image. Furthermore, wavelet analysis can be performed on three-dimensional image stacks to make good images outstanding (Fig. 10).

Wavelet analysis is broadly used to compress images, including images transmitted over the internet and for the rapid searching of fingerprint files. Wavelet analysis is used to enhance inherently low contrast images, such as mammograms, or noisy applications in astronomy. This latter application is very similar to problem of looking for channel opening events in the presence of noise. Wavelet analysis is a general parameter-free procedure to look for objects of varying size scales. As software becomes more accessible in microscopy, applications will include de-noising, image enhancement, image storage and transmission.

\section{FUTURE}

Throughout this review, I have presented practical advise to experimentalists to improve fluorescence imaging as well as new tools or methods such as noise suppression, objectives, labels, photobleaching reduction, and image analysis. In this section I will describe additional cutting edge approaches not discussed above and, in some cases, not yet on the market which will be useful in immunology and cell biology.

\section{Peripheral Instrumentation}

Solid-state light sources are gaining popularity in microscopy applications. In some cases, light emitting diodes (LEDs) are replacing mercury and xenon lamps (Martin et al., 2005; Mazzini et al., 2004). LEDs are semiconductor diodes of various chemical compositions. Their chemical compositions determine the wavelengths of light produced, which varies across the visi- 

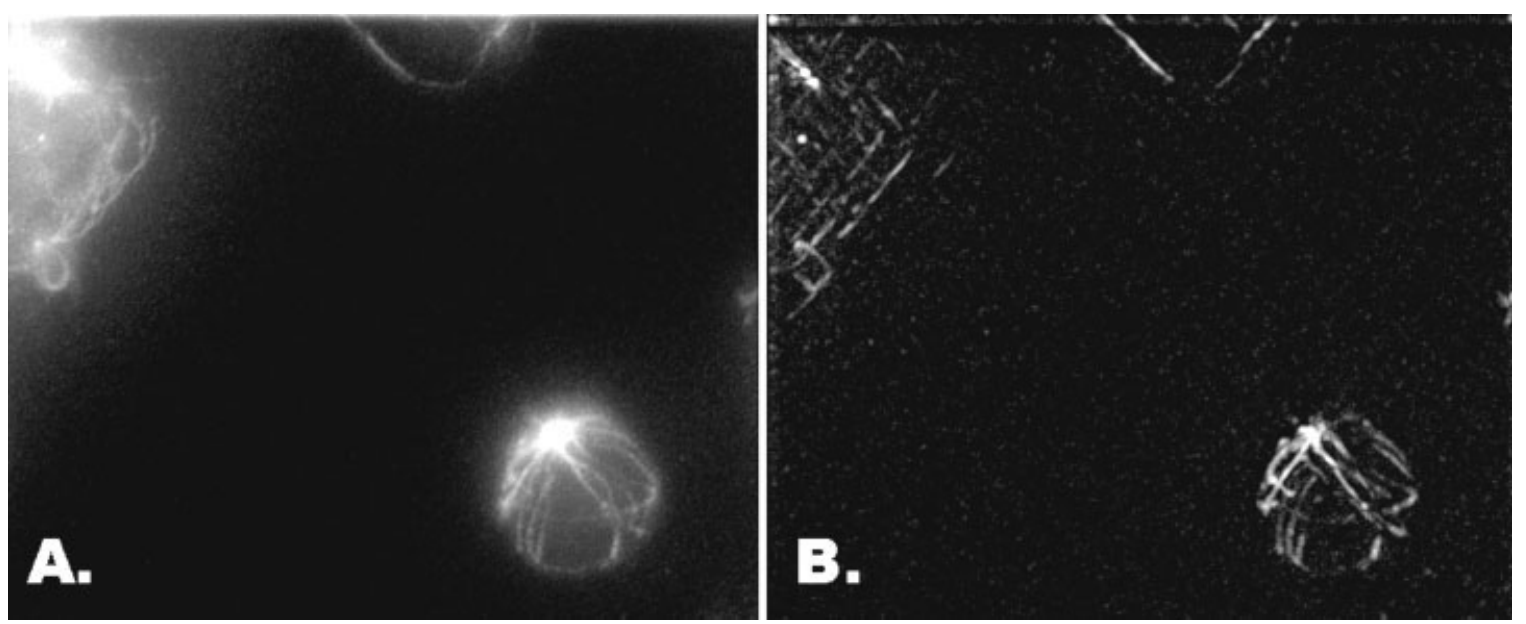

Fig. 10. Imaging leukocyte microtubules. Leukocytes were fixed then labeled with antimicrotubule Abs. Z-scans were performed then deconvoluted using an approximate point spread function. Panel A shows microtubules radiating from the centrosome of a lymphocyte. In panel $\mathbf{B}$ the stack was filtered using wavelet software (data corresponding to one wavelet coefficient is shown).

ble spectrum, but is typically limited to a narrow 15 $\mathrm{nm}$ band of emission. LEDs are very efficient, providing high power at the desired wavelength with a low voltage power supply. Additional advantages of LEDs are that they have a 50,000 h lifetime, produce little heat, and do not need lamp adjustments. The disadvantage is that a different lamp would be required for each excitation wavelength needed in an experiment. Although this may not be a serious limitation for clinical laboratories using only a single wavelength of light, it may be an important limitation in multi-probe labeling experiments. Currently, LEDs are being used more often in fluorescence stereomicroscopes. Although LEDs are generally used in a continuous mode, they can be modulated at $300 \mathrm{MHz}$ (Szmacinski and Chang, 2000), making them a useful alternative in phase-sensitive FLIM (Herman et al., 2001). In addition to LEDs, diode lasers are another solid state illumination device gaining in popularity in the microscope community. In many respects, the performance of diode lasers now match that of many water- or air-cooled gas lasers.

As outlined above, the introduction of EMCCD cameras has increased the number of options available to experimentalists. Use of the CMOS chip architecture in biological imaging should increase in the coming years. These chips are far less expensive than other chips, and are used in conventional home digital cameras and in certain clinical imaging applications needing a large pixel array. Because of on-board electronics, CMOS chips are inherently less sensitive. The time required to download a CMOS chip is negligible in comparison to a CCD chip, which makes high repetition rates possible. Indeed, Dr. Etoh's laboratory at Kinki University in Japan has recently constructed an intensified camera capable of one million frames per second for applications in hydrodynamics. Although this is faster than biological applications demand, it does indicate that download times associated with current imaging protocols can be eliminated to provide rapid-fire dynamics of biological processes.

\section{Emerging Microscopes}

A variety of new microscopes are under development and a few have entered the market recently, such as TIRF, NSOM and 4Pi microscopy. One type of emerging microscope based upon image interference methods is 4Pi microscopy (Egner and Hell, 2005). In 4Pi microscopy, a thin fluorescently labeled sample is held between two coverslips. High numerical aperture objectives located above and below the sample are brought to focus at the same point. As fluorescence is emitted in all directions, two objectives collect more light than just one, thereby improving the lateral and axial resolutions. Two sets of mirrors, beamsplitters, etc. are used to illuminate the sample. In the confocal mode, this is called 4Pi microscopy, but in the wide field mode it is called image interference microscopy $\left(\mathrm{I}^{2} \mathrm{M}\right)$. Light collected by each objective then travels the same distance to interfere at a detector. This provides roughly a 4 -fold improvement in axial resolution $\left(d_{\mathrm{z}}=\right.$ $110 \mathrm{~nm})$ in comparison to confocal microscopy. The Leica TCS 4Pi microscope is now commercially available.

Another important emerging microscope is the stimulated emission depletion (STED) microscope, which was pioneered by Hell and colleagues in Goettingen (Egner and Hell, 2005; Hell and Wichmann, 1994; Klar et al., 2001). Astounding lateral resolutions of $\sim 30 \mathrm{~nm}$ have been obtained with $700 \mathrm{~nm}$ excitation light. In STED microscopy, the spot of a scanning confocal system is reduced in size to increase spatial resolution. As in confocal or two-photon microscopy, a laser pulse at a dye's excitation wavelength excites the fluorescent molecules within the diffraction-limited spot. Before the sample emits, a second laser provides a longer wavelength high power pulse of light to deplete excited probes at the periphery of the original spot. The stimulated emission due to the second laser pulse takes place in a period of time short in comparison to fluorescence emission. The second laser pulse is offset slightly from the original spot or it can be doughnut- 
shaped; in either case, the new spot of excited probes is much smaller than the original diffraction-limited spot, thereby leading to high resolution images of spontaneous fluorescence emission. STED and 4Pi methodologies yield high axial and lateral resolution images. Both approaches are inefficient, but could be very effective when combined with other new labeling technologies such as quantum dots or nanodiamonds. STED fluorescence microscopy has already been used to collect striking immunofluorescence micrographs (Dyba et al., 2003; Hell, 2003).

Gustafsson (2005) has recently demonstrated saturated structured-illumination fluorescence microscopy (SSIM), which provides a lateral resolution of $<50 \mathrm{~nm}$. As described above, structured illumination provides enhanced lateral and axial resolution by converting invisible high resolution information into visible moiré fringes (at least electronically). SSIM extends this approach by introducing harmonics into the pattern, which contain additional higher resolution information. The advantage of SSIM is that its hardware should be a fraction of the cost of 4Pi or STED microscopy. However, in practice, it will require the use of bright, photostable probes or fixed cells treated with inhibitors of photobleaching to yield high resolution images.

\section{CONCLUSIONS}

The applications of fluorescence microscopy in biology have been growing rapidly in recent years and will have an important impact on immunology. Specifically, these fluorescence tools will help to bridge molecular and cellular immunology. The purpose of this review article is to facilitate the transfer of emerging biophysical instrumentation and methodology to immunological problems. This review should improve the fluorescence microscopic skills of graduate students and professionals in immunology seeking to investigate their problems using fluorescence microscopy.

Many people in biology and medicine still believe that optical microscopy is limited by the Rayleigh criterion. This, of course, is no longer the case, as illustrated by the $\mathrm{z}$ axis resolution of scanning confocal and twophoton microscopy. Moreover, commercial 4Pi fluorescence microscopes provide resolutions of $d_{\mathrm{x}, \mathrm{y}} \sim 100 \mathrm{~nm}$ and $d_{\mathrm{z}} \sim 100 \mathrm{~nm}$ while the surface illumination method of NSOM provides a $d_{\mathrm{x}, \mathrm{y}} \geq 20 \mathrm{~nm}$. More specialized techniques such as STED can provide a lateral and axial resolution of $40 \mathrm{~nm}$ without being restricted to surface illumination. Several of these microscopic methods are summarized in Figure 11. It is often the case that as the spatial resolution increases, the system's efficiency decreases, making the extraction of weak signals all the more important.

Although traditional fluorescence microscopy is very mature technique, the innovations in fluorescence microscopy instrumentation, labels, and signal processing tools are growing at a very rapid pace. These techniques, when properly used, have the potential to dramatically impact our understanding of immunology. Scientists will find themselves challenged to keep abreast of new techniques in this rapidly changing field. Using haptens or ligands on surfaces, NSOM and TIRF microscopy will provide novel insights in single molecule studies and ion channel opening events.

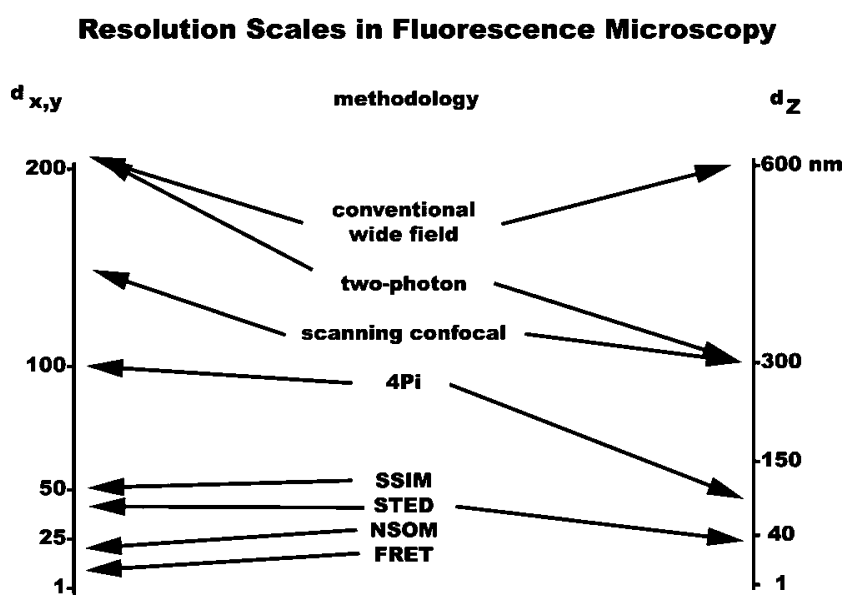

Fig. 11. A survey of the lateral $\left(d_{\mathrm{x}, \mathrm{y}}\right)$ and axial $\left(d_{\mathrm{z}}\right)$ resolutions provided by a variety of fluorescence microscopic tools.

STED fluorescence microscopy has the potential to supply near single molecule resolution data for intact cells using ultra-stable markers, such as quantum dots. Using quantum dot labeling with multiple spectroscopic signatures, it should be possible to study the simultaneous trafficking of various cell populations. In addition to improving the spatial resolution of molecules, it is also possible to improve the temporal resolution of biological events using brief pulses of saturating excitation light during wide field illumination. Using endogenous labels such as GFP, the intricate molecular events accompanying immunological events, such as tumor cell killing, should be revealed.

\section{ACKNOWLEDGMENTS}

I thank members of my research group for collecting several examples noted above.

\section{REFERENCES}

Abbe E. 1873. Beitrage zur theorie des mikroskops und der mikroskopischen wahrnehmug. Schultze Arch Mikr Anat 9:413.

Alivisatos AP, Gu W, Larabell C. 2005. Quantum dots as cellular probes. Ann Rev Biomed Eng 7:55-76.

Axelrod D. 2001. Total internal reflection fluorescence microscopy in cell biology. Traffic 2:764-774.

Balle B, Farkas DL, Taylor DL, Lanni F. 1993. Enhancement of axial resolution in fluorescence microscopy by standing-wave excitation. Nature 366:44-48.

Baschong W, Suetterlin R, Laeng RH. 2001. Control of autofluorescence of archival formaldehyde-fixed, paraffin-embedded tissue in confocal laser scanning microscopy (CLSM). J Histochem Cytochem 49:1565-1571.

Baturin AS, Chadaev NN, Leshukov MY, Sheshin EP. 2003. Application of holographic diffusers to improve light uniformity of source with carbon fiber cathodes. J Vac Sci Technol B 21:1629-1632.

Bernas T, Zarebski M, Cook RR, Dobrucki JW. 2004. Minimizing photobleaching during confocal microscopy of fluorescent probes bound to chromatin: Role of anoxia and photon flux. J Microscopy 215:281-296.

Breeze RH, Ke B. 1972. Some comments on xenon arc lamp stability. Rev Sci Instrum 43:821-823.

Broser PJ, Schulte R, Lang S, Rogh A, Helmchen F, Waters J, Sakmann B, Wittum G. 2004. Nonlinear anisotropic diffusion filtering of three-dimensional image data from two-photon microscopy. J Biomed Opt 9:1253-1264.

Cappello G, Badoual M, Ott A, Prost J, Busoni L. 2003. Kinesin motion in the absence of external forces characterized by interfer- 
ence total internal reflection microscopy. Phys Rev E Stat Nonlin Soft Matter Phys 68:021907-021917.

Chudakov DM, Lukyanov S, Lukyanov KA. 2005. Fluorescent proteins as a toolkit for in vivo imaging. Trends Biotechnol 23:605613.

Clancy B, Cauller LJ. 1998. Reduction of background autofluorescence in brain sections following immersion in sodium borohydride. J Neurosci Methods 83:97-102.

Coates CG, Denvir DJ, McHale NG, Thornbury KD, Hollywood MA. 2004. Optimizing low-light microscopy with back-illuminated electron multiplying charge-coupled device: Enhanced sensitivity, speed, and resolution. J Biomed Opt 9:1244-1252.

Connally R, Veal D, Piper J. 2002. High resolution detection of fluorescently labeled microorganisms in environmental samples using time-resolved fluorescence microscopy. FEMS Microbiol Ecol 41:239-245.

Connally R, Veal D, Pipe J. 2003. Novel flashlamp based timeresolved fluorescence microscope reduces autofluorescence for 30fold contrast enhancement in environmental samples. In: Conchello JA, Cogswell CJ, Wilson T, editors. Three-dimensional and multidimensional microscopy: Image acquisition and processing $\mathrm{X}$, Vol. 4964. Proceedings of the SPIE. pp 14-23.

Connally R, Veal D, Piper J. 2004. Flash lamp-excited time-resolved fluorescence microscope suppresses autofluorescence in water concentrates to deliver an 11-fold increase in signal-to-noise ratio. J Biomed Opt 9:725-734

Cummins CM, Koivunen ME, Stephanian A, Gee SJ, Hammock BD, Kennedy IM. 2006. Application of europium(III) chelate-dyed nanoparticle labels in a competitive atrazine fluoroimmunoassay on an ITO waveguide. Biosens Bioelectron 21:1077-1085.

de Haas RR, van Gijlswijk RP, van der Tol EB, Zijlmans HJ, BakkerSchut T, Bonnet J, Verwoerd NP, Tanke HJ. 1997. Platinum porphyrins as phosphorescent label for time-resolved microscopy. J Histochem Cytochem 45:1279-1292.

Demuro A, Parker I. 2005. Optical single-channel recording: Imaging $\mathrm{Ca}^{2+}$ flux through individual ion channels with high temporal and spatial resolution. J Biomed Opt 10:11002-11008.

Denvir DJ, Conroy E. 2002. Electron multiplying CCD technology: The new ICCD. Presented at SPIE Annual Meeting, Seattle, July.

Dittrich PS, Schwille P. 2001. Photobleaching and stabilization of fluorophores used for single-molecule analysis with one- and two-photon excitation. Appl Phys B 73:829-837.

Dosev D, Nichkova M, Liu M, Guo B, Liu GY, Hammock BD, Kennedy IM. 2005. Application of luminescent $\mathrm{Eu}: \mathrm{Gd}(2) \mathrm{O}(3)$ nanoparticles to the visualization of protein micropatterns. J Biomed Opt 10:64006.

Dussault D, Hoess P. 2004. ICCDs edge out electron-multiplying CCDs in low light. Laser Focus World 40:69-76.

Dyba M, Jakobs S, Hell SW. 2003. Immunofluorescence stimulated emission depletion microscopy. Nat Biotechnol 21:1303-1304.

Eggeling C, Eolkmer A, Seidel CAM. 2005. Molecular photobleaching kinetics of rhodamine $6 \mathrm{G}$ by one- and two-photon induced confocal fluorescence microscopy. Chem Phys Chem 6:791-804.

Egner A, Hell SW. 2005. Fluorescence microscopy with super-resolved optical sections. Trends Cell Biol 15:207-215.

French T, So PT, Weaver DJ, Jr, Coelho-Sampaio T, Gratton E, Voss EW, Jr, Carrero J. 1997. Two-photon fluorescence lifetime imaging microscopy of macrophage-mediated antigen processing. J Microsc 185:339-353.

Gao X, Yang L, Petros JA, Marshall FF, Simons JW, Nie S. 2005. In vivo molecular and cellular imaging with quantum dots. Curr Opin Biotechnol 16:63-72.

Goldman RD, Spector DL. 2005. Live cell imaging: A laboratory manual. Cold Spring Harbor, NY: Cold Spring Harbor Laboratory Press.

Grayson MH, Hotchkiss RS, Karl IE, Holtzman MJ, Chaplin DD. 2003. Intravital microscopy comparing $\mathrm{T}$ lymphocyte trafficking to the spleen and the mesenteric lymph node. Am J Physiol Heart Circ Physiol 284:H2213-H2226.

Greenbaum L, Rothmann C, Lavie R, Malik Z. 2000. Green fluorescent protein photobleaching: A model for protein damage by endogenous and exogenous singlet oxygen. Biol Chem 381:1251-1258.

Griesbeck O. 2004. Fluorescent proteins as sensors for cellular functions. Curr Opin Neurobiol 14:636-641.

Gustafsson MG. 2005. Nonlinear structured-illumination microscopy: Wide-field fluorescence imaging with theoretically unlimited resolution. Proc Natl Acad Sci USA 102:13081-13086.

Gustafsson MGL. 2000. Surpassing the lateral resolution limit by a factor of two using structured illumination microscopy. J Microscopy 198:82-87.

Gustafsson MGL, Agard DA, Sedat JW. 1999. I ${ }^{5}$ M: 3D widefield light microscopy with better than $100 \mathrm{~nm}$ axial resolution. J Microsc 195:10-16.
Hafeman DG, Seul M, Cliffe II CM, McConnell HD. 1984. Superoxide enhances photobleaching during cellular immune attach against fluorescent lipid monolayer membranes. Biochim Biophys Acta 772:20-28.

Hasan MT, Friedrich RW, Euler T, Larkum ME, Giese G, Both M, Duebel J, Waters J, Bujard H, Griesbeck O, Tsien RY, Nagai T, Miyawaki A, Denk W. 2004. Functional fluorescent $\mathrm{Ca}^{2+}$ indicator proteins in transgenic mice under TET control. PLOS Biol 2:763-775.

Hell SW. 2004. Toward fluorescence nanoscopy. Nat Biotechnol 21:1347-1355.

Hell SW, Wichmann J. 1994. Breaking the diffraction resolution limit by stimulated emission: Stimulated-emission-depletion fluorescence microscopy. Opt Lett 19:780-782.

Herman P, Maliwal BP, Lin H-J, Lakowicz JR. 2001. Frequency-domain fluorescence microscopy with the LED as a light source. J Microsc 203:176-181.

Herrero FA. 1992. Light-trap design using multiple reflections and solid-angle attenuation: Application to a spaceborne electron spectrometer. Appl Opt 31:5331-5340.

Hynecek J, Nishiwaki T. 2003. Excess noise and other important characteristics of low light level imaging using charge multiplying CCDs. IEEE Trans Electronic Dev 50:239-245.

Inoue S. 1989. Video enhancement and image processing in light microscopy. Part 1: Video microscopy. Am Lab April:52-60.

Inoue S, Inoue T. 1989. Video enhancement and image processing in light microscopy. Part 2: Digital image processing. Am Lab April:62-81.

Inoue S, Spring KR. 1997. Video microscopy. New York: Plenum Press. Chapter 13.

Jaiswal JK, Simon SM. 2004. Potentials and pitfalls of fluorescent quantum dots for biological imaging. Trends Cell Biol 14:497-504.

Johnson GD, de Nogueira C, Araujo GM. 1981. A simple method of reducing the fading of immunofluorescence during microscopy. J Immunol Methods 43:349-350.

Johnson GD, Davidson RS, McNamee KC, Russel G, Goodwin D, Holborow EJ. 1982. Fading of immunofluorescence during microscopy: A study of the phenomenon and its remedy. J Immunol Methods 55:231-242.

Klar TA, Engel E, Hell SW. 2001. Breaking Abbe's diffraction resolution limit in fluorescence microscopy with stimulated emission depletion beams of various shapes. Phys Rev E 64:066613-1/ 066613-9.

Kindzelskii AL, Sitrin RG, Petty HR. 2004. Optical microspectrophotometry supports the existence of gel phase lipid rafts at the lamellipodium of neutrophils: Apparent role in calcuim signaling. J Immunol 172:4681-4685.

Koizumi M, Usui Y. 1972. Fundamental aspects of oxidative and reductive photobleaching of xanthene and thiazine dyes. Mol Photochem 4:57-92.

Kong SK, Ko S, Lee CY, Lui PY. 1999. Practical considerations in acquiring biological signals from confocal microscopy. Methods Enzymol 307:20-26.

Koopman M, Cambi A, de Bakker BI, Joosten B, Figdor CG, van Hulst NF, Garcia-Parajo MF. 2004. Near-field scanning optical microscopy in liquid for high resolution single molecule detection on dendritic cells. FEBS Lett 573:6-10.

Lakowicz ,JR. 1983. Principles of fluorescence spectroscopy. New York: Plenum.

Lee MF, Kong SK, Fung KP, Lui CP, Lee CY. 1996. Practical considerations in acquiring biological signals from confocal microscope: Solvent effect and temperature effect. Biol Signals 5:291-300.

Legg JW, Lewis CA, Parsons M, Ng T, Isacke CM. 2002. A novel PKCregulated mechanism controls CD44 ezrin association and directional cell motility. Nat Cell Biol 4:399-407.

Lewis A, Taha H, Strinkovski A, Manevitch A, Khatchatouriants A, Dekhter R, Ammann E. 2003. Near-field optics: From subwavelength illumination to nanometric shadowing. Nat Biotech 21 : 1378-1386.

Lui PPY, Lee MMF, Ko S, Lee CY, Kong SK. 1997. Practical considerations in acquiring biological signals from confocal microscope. II. Laser-induced rise of fluorescence and effect of agonist droplet application. Biol Signals 6:45-51.

Liu T, Liu B, Zhang H, Wang Y. 2005. The fluorescence bioassay platforms on quantum dots nanoparticles. J Fluoresc 15:729-733.

Magnan P. 2003. Detection of visible photons in CCD and CMOS: A comparative view. Nucl Instrum Methods Phys Res A 504:199-212.

Mathies RA, Peck K, Stryer L. 1990. Optimization of high-sensitivity fluorescence detection. Anal Chem 62:1786-1791.

Martin G, Agostini HT, Hansen LL. 2005. Light emitting diode microscope illumination for green fluorescent protein isothiocynate epifluorescence. Bio Tech 38:204-206. 
Mazzini G, Ferrari C, Baraldo N, Mazzini M, Angelini M. 2004. Improvements in fluorescence microscopy allowed by high power light emitting diodes. Curr Issues Multidis Microsc Res Educ 181:8.

Meaburn J. 1966. The stability of interference filters. Appl Opt 5:1757-1759.

Michalet X, Pinaud FF, Bentolila LA, Tsay JM, Doose S, Li JJ, Sundaresan G, Wu AM, Gambhir SS, Weiss S. 2005. Quantum dots for live cells, in vivo imaging, and diagnostic. Science 307:538-544.

Minta A, Kao JP, Tsien RY. 1989. Fluorescent indicators for cytosolic calcium based on rhodamine and fluorescein chromophores. J Biol Chem 264:8171-8178.

Miyawaki A, Llopis J, Heim R, McCaffery JM, Adams JA, Ikura M, Tsien RY. 1997. Fluorescent indicators for $\mathrm{Ca}^{2+}$ based on green fluorescent proteins and calmodulin. Nature 388:882-887.

Moss WC, Haase S, Lyle M, Agard DA, Sedat JW. 2005. A novel 3D wavelet-based filter for visualizing features in noisy biological data. J Microscopy 219:43-49.

Neill MAA, Juskaitis R, Wilson T. 1997. Method of obtaining optical sectioning by using structured light in a conventional microscope. Opt Lett 22:1905-1907.

Neumann M, Gabel D. 2002. Simple method for reduction of autofluorescence in fluorescence microscopy. J Histochem Cytochem 50:437-439.

Nichkova M, Dosev D, Perron R, Gee SJ, Hammock BD, Kennedy IM 2006. $\mathrm{Eu}(3+)$-doped $\mathrm{Gd}(2) \mathrm{O}(3)$ nanoparticles as reporters for optical detection and visualization of antibodies patterned by microcontact printing. Anal Bioanal Chem 384:631-637.

Nishimura M, Shirasawa H, Song WJ. 2006. A light-emitting diode light source for imaging of neural activities with voltage-sensitive dyes. Neurosci Res 54:230-234.

Ober RJ, Martinez C, Lai X, Zhou J, Ward ES. 2004. Exocytosis of IgG as mediated by the receptor, FcRn: An analysis at the single-molecule level. Proc Natl Acad Sci USA 101:11076-11081.

Ono M, Murakami T, Kudo A, Isshiki M, Sawada H, Segawa A. 2001. Quantitative comparison of anti-fading mounting media for confocal laser scanning microscopy. J Histochem Cytochem 49:305-311.

Panchuk-Voloshina N, Haugland RP, Bishop-Steward J, Bhalgat MK Millard PJ, Mao F, Leung W-Y, Haugland RP. 1999. Alexa dyes, series of new fluorescent dyes that yield exceptionally bright, photostable conjugates. J Histochem Cytochem 47:1179-1188.

Patterson GH, Piston DW. 2000. Photobleaching in two-photon excitation microscopy. Biophys J 78:2159-2162.

Perona P, Malik J. 1990. Scale-space and edge detection using anisotropic diffusion. IEEE Trans Pattern Anal Mach Intell 12:629-639.

Piston DW. 1998. Choosing objective lenses: The importance of numerical aperture and magnification in digital microscopy. Biol Bull 195:1-4.

Pollack SA. 1966. Angular dependence of transmission characteristics of interference filters and application to a tunable fluorometer. App Opt 5:1749-1756.

Pologruto TA, Yasuda R, Svoboda K. 2004. Monitoring neural activity and $\left[\mathrm{Ca}^{2+}\right]$ with genetically encoded $\mathrm{Ca} 2+$ indicators. J Neurosci 24:9572-9579.

Renikuntla BR, Rose HC, Eldo J, Waggoner AS, Armitage BA. 2004. Improved photostability and fluorescence properties through polyfluorination of a cyanine dye. Org Lett 6:909-912.

Rogers KL, Stinnakre J, Agulhon C, Jublot D, Shorte SL, Kremer EJ, Brulet P. 2005. Visualization of local $\mathrm{Ca}^{2+}$ dynamics with genetically encoded bioluminescent reporters. Eur J Neurosci 21:597610.

Rulli M, Kuusisto A, Salo J, Kojola H, Simell O. 1997. Time-resolved fluorescence imaging in islet cell autoantibody quantitation. J Immunol Methods 208:169-179.

Rumyantsev SL, Shur MS, Bilenko Y, Kosterin PV, Salzberg BM. 2004. Low frequency noise and long-term stability of noncoherent light sources. J Appl Phys 96:966-969.

Sabri S, Richelme F, Pierres A, Benoliel A-M, Bongrand P. 1997. Interest of image processing in cell biology and immunology. J Immunol Methods 208:1-27.

Safiejko-Mroczka B, Bell PB, Jr. 1996. Bifunctional protein cross-linking reagents improve labeling of cytoskeletal proteins for qualitative and quantitative fluorescence microscopy. J Histochem Cytochem 44:641-656.

Salzberg BM, Grinvald A, Cohen LB, Davila HV, Ross WN. 1977. Optical recording of neuronal activity in an invertebrate cenetral nervous system: Simultaneous monitoring of several neurons. J Neurophysiol 40:1281-1291.

Schafer-Hales KJ, Belfield KD, Yao S, Frederiksen PK, Hales JM, Kolattukudy PE. 2005. Fluorene-based fluorescent probes with high two-photon action cross-sections for biological multiphoton imaging applications. J Biomed Opt 10:51402-51408.

Shaner NC, Steinbach PA, Tsien RY. 2005. A guide to choosing fluorescent proteins. Nat Methods 2:905-910.

Smith AM, Gao X, Nie S. 2004a. Quantum dot nanocrystals for in vivo molecular and cellular imaging. Photochem Photobiol 80:377-385.

Smith N, Coates C, Giltinan A, Howard J, O'Connor A, O'Driscoll S, Hauser M, Wagner S. 2004b. EMCCD technology and its impact on rapid low-light photometry. In: Garnett J, Beletic JW, editors. Optical and Infrared Detectors for Astronomy, Vol. 5499. Proceedings of SPIE. pp 162-172.

Song L, Hennink EJ.Young IT, Tanke HJ. 1995. Photobleaching kinetics of fluorescein in quantitative fluorescence microscopy. Biophys J 68:2588-2600.

Song L, Varma CAGO, Verhoeven JW, Tanke HJ. 1996. Influence of the triplet excited state on the photobleaching kinetics of fluorescein in microscopy. Biophys J 70:2959-2968.

Song L, van Gijlswijk RPM, Young IT, Tanke HJ. 1997. Influence of fluorochrome labeling density on the photobleaching kinetics of fluorescein in microscopy. Cytometry 27:213-223.

Sonoda KH, Nakao S, Nakamura T, Oshima T, Qiao H, Hisatomi T, Kinoshita S, Ishibashi T. 2005. Cellular events in the normal and inflamed cornea. Cornea 24 (Suppl 8):S50-S54.

Srivastava M, Jung S, Wilhelm J, Fink L, Buhling F, Welte T, Bohle RM, Seeger W, Lohmeyer J, Maus UA. 2005. The inflammatory versus constitutive trafficking of mononuclear phagocytes into the alveolar space of mice is associated with drastic changes in their gene expression profiles. J Immunol 175:1884-1893.

Steinkamp JA, Lehnert NM, Keij JF, Lehnert BE. 1999. Enhanced immunofluorescence measurement resolution of surface antigens on highly autofluroescent, glutaraldehyde-fixed cells analyzed by phase-sensitive flow cytometry. Cytometry 37:275-283.

Stern MD, Spurgeon HA, Hansford R, Lakatta EG Capogrossi MC. 1989. Optimum spectral windows to minimize quantum noise of ratiometric intracellular fluorescent probes. Cell Calcium 10:527-534.

Stewart CN, Jr. 2006. Go with the glow: Fluorescent proteins to light transgenic organisms. Trends Biotechnol 24:155-162.

Suhling K, French PM, Phillips D. 2005. Time-resolved fluorescence microscopy. Photochem Photobiol Sci 4:13-22.

Szmacinski H, Chang Q. 2000. Micro- and sub-nanosecond lifetime measurements using a UV light-emitting diode. Appl Spectrosc 1:106-109.

Taylor DL, Wang YL, Heiple JM. 1980. Contractile basis of ameboid movement. VII. The distribution of fluorescently labeled actin in living amebas. J Cell Biol 86:590-598.

Theuwissen A, Roks E 2001. Building a better mousetrap: Modified CMOS processes improve image sensor performance. OE Magazine, January 29-32.

Treanor B, Lanigan PM, Suhling K, Schreiber T, Munro I, Neil MA, Phillips D, Davis DM, French PM. 2005. Imaging fluorescence lifetime heterogeneity applied to GFP-tagged MHC protein at an immunological synapse. J Microsc 217:36-43.

Unser M, Aldroubi A. 1996. A review of wavelets in biomedical applications. Proc IEEE 84:626-638.

Uttenweiler D, Weber C, Jahne B, Fink RHA, Scharr H. 2003. Spatiotemporal anisotropic diffusion filtering to improve signal-to-noise ratios and object restoration in fluorescence microscopic image sequences. J Biomed Opt 8:40-47.

Wallrabe H, Periasamy A. 2005. Imaging protein molecules using FRET and FLIM microscopy. Curr Opin Biotechnol 16:19-27.

Yang M, Baranov E, Wang JW, Jiang P, Wang X, Sun FX, Bouvet M, Moossa AR, Penman S, Hoffman RM. 2002. Direct external imaging of nascent cancer, tumor progression, angiogenesis, and metastasis on internal organs in the fluorescent orthotopic model. Proc Nat Acad Sci USA 99:3824-3829.

Yu S-J, Kang M-W, Chang H-C, Chen K-M, Yu Y-C. 2005. Bright fluorescent nanodiamonds: No photobleaching and low cytoxicity. J Am Chem Soc 127:17604-17605.

Zakrzewski J. 2003. Integrating a spectrometer with an optical microscope presents challenges. SPIE OE Magazine, November. p 29

Zal T, Gascoigne NR. 2004. Using live FRET imaging to reveal early protein-protein interactions during $\mathrm{T}$ cell activation. Curr Opin Immunol 16:674-683.

Zhao M, Yang M, Baranov E, Wang X, Penman S, Moossa AR, Hoffman RM. 2001. Spatial-temporal imaging of bacterial infection and antibiotic response in intact animals. Proc Natl Acad Sci USA 98:9814-9818 